\title{
AFFINE MANIFOLDS AND ORBITS OF ALGEBRAIC GROUPS ${ }^{1}$
}

\author{
WILLIAM M. GOLDMAN AND MORRIS W. HIRSCH \\ Dedicated to the memory of Jacques Vey
}

\begin{abstract}
This paper is the sequel to The radiance obstruction and parallel forms on affine manifolds (Trans. Amer. Math. Soc. 286 (1984), 629-649) which introduced a new family of secondary characteristic classes for affine structures on manifolds. The present paper utilizes the representation of these classes in Lie algebra cohomology and algebraic group cohomology to deduce new results relating the geometric properties of a compact affine manifold $M^{n}$ to the action on $\mathbf{R}^{n}$ of the algebraic hull $\mathbf{A}(\Gamma)$ of the affine holonomy group $\Gamma \subseteq \operatorname{Aff}\left(\mathbf{R}^{n}\right)$.

A main technical result of the paper is that if $M$ has a nonzero cohomology class represented by a parallel $k$-form, then every orbit of $\mathbf{A}(\Gamma)$ has dimension $\geq k$. When $M$ is compact, then $\mathbf{A}(\Gamma)$ acts transitively provided that $M$ is complete or has parallel volume; the converse holds when $\Gamma$ is nilpotent. A 4-dimensional subgroup of $\operatorname{Aff}\left(\mathbf{R}^{3}\right)$ is exhibited which does not contain the holonomy group of any compact affine 3-manifold.

When $M$ has solvable holonomy and is complete, then $M$ must have parallel volume. Conversely, if $M$ has parallel volume and is of the homotopy type of a solvmanifold, then $M$ is complete. If $M$ is a compact homogeneous affine manifold or if $M$ possesses a rational Riemannian metric, then it is shown that the conditions of parallel volume and completeness are equivalent.
\end{abstract}

This paper is the sequel to our previous paper [22]. In that paper we exploited certain characteristic classes (exterior powers of the radiance obstruction) to obtain relationships between various properties of affine manifolds. Our results supported the conjecture (first made by L. Markus [36]) that a compact affine manifold is complete if and only if it has parallel volume. We shall refer to this as the main conjecture.

Let $M$ be a compact affine manifold with developing map dev: $\tilde{M} \rightarrow \mathbf{E}$ and affine holonomy representation $h: \pi \rightarrow h(\pi)=\Gamma \subset \operatorname{Aff}(\mathbf{E})$. Here $\pi$ is the group of deck transformations of the universal cover $\tilde{M}, \mathbf{E}$ is the vector space $\mathbf{R}^{n}$, and $\operatorname{Aff}(\mathbf{E})$ is the group of affine automorphisms of $\mathbf{E}$. The map dev is a locally affine immersion which is equivariant respecting $h$. The linear holonomy homomorphism is the composition $\lambda: \pi \rightarrow \mathrm{GL}(\mathbf{E})$ of $h$ with the natural homomorphism $\mathbf{L}: \operatorname{Aff}(\mathbf{E}) \rightarrow \operatorname{GL}(\mathbf{E})$. The obstruction to $\Gamma$ fixing a point in $\mathbf{E}$ is a 1-dimensional cohomology class $c_{M} \in H^{1}\left(M ; \mathbf{E}_{\lambda}\right)$ with coefficients in $\mathbf{E}$ twisted by $\lambda$. It comes from a universal class in the group cohomology $H^{1}\left(\operatorname{Aff}(\mathbf{E}) ; \mathbf{E}_{L}\right)$ which contains the crossed homomorphism $u: \operatorname{Aff}(\mathbf{E}) \rightarrow \mathbf{E}, g \mapsto g(0)$.

Received by the editors December 12, 1984 and, in revised form, May 29, 1985.

1980 Mathematics Subject Classification (1985 Revision). Primary 57R99, 53C05; Secondary 53C10, 55R25.

${ }^{1}$ Research partially supported by fellowships and grants from the National Science Foundation. 
The results in our previous paper were obtained by calculating $c_{M}$ in various cohomology theories - singular, Cech, and de Rham. In the present work we obtain further information by relating $c_{M}$ to the cohomology of the algebraic hull $\mathbf{A}(\Gamma)$ of $\Gamma$ in $\operatorname{Aff}(\mathbf{E})$. Our general theme is to relate geometrical properties of $M$ to algebraic properties of $\mathbf{A}(\Gamma)$. For example the main conjecture states that completeness, a geometrical property of $M$, is equivalent to the condition that $\mathbf{A}(\Gamma)$ preserve volume in $\mathbf{E}$.

Many other properties of $M$ can be described in terms of $\mathbf{A}(\Gamma)$. Thus $\Gamma$ is solvable when the algebraic group $\mathbf{A}(\Gamma)$ has the same property. It turns out that this condition brings us closer to affirming the main conjecture.

Proposition S. Let $M$ be a compact affine manifold with solvable holonomy group $\Gamma$. Then:

(a) If $M$ is complete, then $M$ has parallel volume.

(b) If $M$ has parallel volume and the solvable rank of $\Gamma$ is less than the dimension of $M$, then $M$ is complete.

(The solvable rank is the minimum sum of the ranks of the abelian quotients in a composition series. For example, the hypotheses of (b) are satisfied if $M$ is homotopy-equivalent to a solvmanifold.)

One of the main components of the proof of Proposition $\mathrm{S}$ is the following result:

Proposition T. Let $M$ be a compact affine manifold. If $M$ is complete, or if $M$ has parallel volume, then $\mathbf{A}(\Gamma)$ acts transitively on $\mathbf{E}$.

As an immediate corollary we obtain

Proposition F. If $M$ is as in Proposition $\mathrm{T}$ and is connected, then every rational function on $M$ is constant.

By a rational function on $M$ we mean a function defined in a dense open set, which appears rational in every affine coordinate chart. Equivalently, such a function corresponds via the developing map (from the universal covering of $M$ to $\mathbf{E}$ ) to a rational function on $\mathbf{E}$ which is fixed by $\mathbf{A}(\Gamma)$. In a similar way one defires polynomial functions (and more generally tensors) on $M$. It is unknown whether any compact affine manifold can support a nonconstant polynomial function. In dimension three this cannot happen (D. Fried [9], W. Goldman [19]).

For certain classes of affine manifolds, transitivity of $\mathbf{A}(\Gamma)$ is sufficient for completeness. These include homogeneous affine manifolds, manifolds admitting rational Riemannian metrics, and, more generally, affine manifolds whose developing maps are covering maps onto semialgebraic open sets.

Our methods give a geometric proof of the following result of J. Helmstetter [25], which is related to the main conjecture:

Proposition H. A left-invariant affine structure on a Lie group $G$ is complete if and only if every right-invariant volume form on $G$ is parallel.

Our results also apply to manifolds which are not assumed to be complete or to have parallel volume. The basic strategy is to derive a lower bound for the dimensions of the orbits of $\mathbf{A}(\Gamma)$ from knowledge of some cohomologically nontrivial exterior form on the manifold; a parallel volume form is a special case. When $\Gamma$ is nilpotent we use this method to strengthen some of the results of Fried, Goldman and Hirsch $[\mathbf{1 3}]$ : 
PROPOSITION N. Let $M$ be a compact affine manifold with nilpotent holonomy group $\Gamma$. Then:

(a) The highest degree of a nonvanishing exterior power of the radiance obstruction equals the smallest dimension of an orbit of $\mathbf{A}(\Gamma)$.

(b) $M$ is complete if and only if $\mathbf{A}(\Gamma)$ acts transitively on $\mathbf{E}$.

(c) If $M$ is incomplete, then the orbits of $\mathbf{A}(\Gamma)$ having smallest dimension lie outside the developing image (in fact there is only one).

It seems reasonable to conjecture that a similar picture holds more generally, say for $\Gamma$ solvable. This has been verified for the special case that $M$ has dimension three and solvable fundamental group (Goldman [19]).

By explicit example we show that condition (a) of Proposition $\mathrm{N}$ does not hold for arbitrary algebraic subgroups of the affine group. In this way condition (a) can be used to exclude certain subgroups of $\mathrm{Aff}(\mathbf{E})$ from being holonomy groups of compact affine manifolds. More precisely we exhibit algebraic subgroups of $\operatorname{Aff}(\mathbf{E})$ which act transitively on $\mathbf{E}$ and do not contain any such holonomy groups.

The proofs of these results heavily use the structure theory of nilpotent affine groups which is developed in our previous paper [13]. The unique smallest-dimensional orbit of the algebraic hull is proved to be an affine subspace $\mathbf{E}_{U}$, coinciding with what we called the Fitting subspace in [13]. This subspace is characterized as the unique $\Gamma$-invariant affine subspace upon which $\Gamma$ acts unipotently (i.e. the linear part $\mathbf{L}(\Gamma) \subset \mathrm{GL}(\mathbf{E})$ is a group of unipotent linear transformations). The dimension of $\mathbf{E}_{U}$ is a measure of completeness of the affine structure, because the corresponding power of the radiance obstruction is a cohomological invariant whose nonvanishing expresses an algebraic condition akin to parallel volume. We shall call the restriction of $\Gamma$ to $\mathbf{E}_{U}$ the Fitting component of the holonomy group of $M$. A necessary condition that a unipotent affine action of a finitely generated group $G$ be a Fitting component of the holonomy of a compact manifold is that the action be syndetic on all of $\mathbf{E}$, i.e. that there exists a compact set $K \subset \mathbf{E}$ such that $G K=\mathbf{E}$. Not every syndetic unipotent affine action can be realized as the Fitting component of a nilpotent holonomy group of a compact affine manifold:

PROPOSITION E. Let $G$ be the subgroup of $\operatorname{Aff}\left(\mathbf{R}^{3}\right)$ comprising the following affine automorphisms:

$$
\left[\begin{array}{lll}
1 & t & u \\
0 & 1 & 2 v \\
0 & 0 & 1
\end{array}\right]\left[\begin{array}{c}
s \\
t+v^{2} \\
v
\end{array}\right]
$$

(where the matrices represent respectively the linear and translational parts of an affine automorphism of $\mathbf{R}^{3}$ ). Then $G$ acts transitively on $\mathbf{R}^{3}$ and does not contain the Fitting component of a nilpotent holonomy group of any compact affine 3-manifold. In particular $G$ does not contain the holonomy group of any compact affine 3-manifold.

(It follows that the finitely generated discrete subgroup consisting of integral matrices does not contain the holonomy group of any such manifold.)

The outline of this paper is as follows. $\S 1$ is an exposition of the cohomology theory of Lie algebras, Lie groups and algebraic groups as it relates to affine actions and radiance. The main result we use is a theorem of Hochschild [29] which 
identifies the algebraic cohomology of an algebraic group as the space of invariants (under a Levi subgroup) in the Lie algebra cohomology of its unipotent radical. From this we deduce our main technical result:

Proposition G. Let $G$ be an algebraic subgroup of $\mathrm{Aff}(\mathbf{E})$. For $x \in \mathbf{E}$, the dimension of the $G$-orbit of $x$ bounds from above the degrees of nonvanishing powers of the radiance obstruction of $G$.

In an appendix to $\S 1$ we give an account of left-invariant affine structures on Lie groups. While most of the results detailed there are known in more general contexts by the work of Helmstetter and others, our treatment is considerably more geometric. We have included these results since they provide beautiful examples of affine structures, whose homogeneity makes them particularly understandable.

$\S 2$ resumes the study of compact affine manifolds. Using Hochschild's theorem, we show how parallel volume implies the transitivity of the algebraic hull. From this we deduce several corollaries: Markus' conjecture for affine structures which are homogeneous or have rational Riemannian metrics, nonexistence of rational functions, upper bounds on the degrees of polynomial tensors, etc. The basic fact we use is the following corollary of Proposition G:

PROPOSITION M. Let $M$ be an affine manifold. If there exists a parallel $k$ form on $M$ which has nonzero cohomology class, then every orbit of the action of the algebraic hull $\mathbf{A}(\Gamma)$ on $\mathbf{E}$ has dimension $\geq k$.

$\S \S 3$ and 4 discuss affine manifolds with solvable and nilpotent holonomy respectively. The partial results on Markus' conjecture use a well-known (but not as well-documented) lemma on representations of solvable groups, which may be found in [11] (which is based upon the treatment in Raghunathan [42]). The results in $\S 4$ on nilpotent holonomy depend on previous results proved in [13 and 23].

ACKNOWLEDGEMENTS. This paper has its roots in our collaboration with D. Fried, which in turn is based upon unpublished work of J. Smillie. We are grateful to them for many helpful conversations. Discussions with G. Hochschild and C. Moore on algebraic groups have been crucial to this work. We are grateful to J. Helmstetter, and the late J. Vey for helpful suggestions and for describing their results to us. Conversations with G. Levitt on foliations have also played an important role in the development of the ideas here.

1. Affine representations of Lie groups, Lie algebras and algebraic groups.

1.1 As usual $\mathbf{E}$ is a finite-dimensional real vector space and GL(E) (respectively $\operatorname{Aff}(\mathbf{E})$ ) is the group of all linear (resp. affine) automorphisms of $\mathbf{E}$. The Lie algebra $\operatorname{gl}(\mathbf{E})$ of $\mathrm{GL}(\mathbf{E})$ consists of all linear vector fields $\sum a_{i j} x_{i} \partial / \partial x_{j}$, while the Lie algebra aff(E) of Aff(E) consists of affine vector fields $\sum\left(a_{i j} x_{i}+b_{j}\right) \partial / \partial x_{j}\left(a_{i j}, b_{j} \in \mathbf{R}\right)$. The canonical homomorphism L: Aff $(\mathbf{E}) \rightarrow \mathrm{GL}(\mathbf{E})$ induces a Lie algebra homomorphism, denoted $\mathbf{L}: \operatorname{aff}(\mathbf{E}) \rightarrow \operatorname{gl}(\mathbf{E})$, whose kernel consists precisely of the parallel vector fields $\sum b_{j} \partial / \partial x_{j}$. If $X \in \operatorname{aff}(\mathbf{E})$ as above, then the translational part $u(X)$ is the unique parallel vector field which agrees with $X$ at 0 , namely $\sum b_{j} \partial / \partial x_{j}$.

1.2 Let $\mathfrak{g}$ be a Lie algebra. Any homomorphism $\rho: \mathfrak{g} \rightarrow \operatorname{gl}(\mathbf{E})$ gives $\mathbf{E}$ the structure of a $\mathfrak{g}$-module. To any $\mathfrak{g}$-module $E$ are associated cohomology groups $H^{*}(\mathfrak{g}, E)$. (See Cartan and Eilenberg [6], Chevalley and Eilenberg [7], and Koszul [33] for more 
details on Lie algebra cohomology.) If $G$ is a Lie group with Lie algebra $\mathfrak{g}$, then the cohomology of $\mathfrak{g}$ is the cohomology of the complex of left-invariant differential forms on $G$. In particular the exterior algebra $\Lambda^{*}\left(\mathfrak{g}^{*}\right)$ on the dual of $\mathfrak{g}$ may be identified with the differential graded algebra of all left-invariant exterior forms on $G$; the resulting cohomology is $H^{*}(\mathfrak{g})$. Now suppose that $\rho: \mathfrak{g} \rightarrow \operatorname{gl}(\mathbf{E})$ defines a $\mathfrak{g}$-module $\mathbf{E}$ (or $\mathbf{E}_{\rho}$ ). Consider the trivial $\mathbf{E}$-bundle over $G$ with a left-invariant flat connection given by

$$
\nabla_{\rho}: \mathbf{E} \rightarrow \mathfrak{g}^{*} \otimes \mathbf{E}, \quad \nabla_{\rho}(v): x \mapsto \rho(x)(v),
$$

where $v \in \mathbf{E}, x \in \mathfrak{g}$. This map extends to a covariant differential operator from exterior $p$-forms with values in $\mathbf{E}$ to exterior $(p+1)$-forms with values in $\mathbf{E}$. The cohomology $H^{*}(\mathfrak{g}, \mathbf{E})$ is defined as the cohomology of the subcomplex comprising left-invariant E-valued differential forms. One can identify this complex with $\bigwedge^{*}(\mathfrak{g}) \otimes \mathbf{E}$, thereby obtaining a differential graded structure on $\Lambda^{*}(\mathfrak{g}) \otimes \mathbf{E}$, with the usual formula for the differential.

1.3 Let $\alpha: \mathfrak{g} \rightarrow \operatorname{aff}(\mathbf{E})$ be an affine representation of the Lie algebra $\mathfrak{g}$. The composition $\mathbf{L} \circ \alpha$ turns $\mathbf{E}$ into a $\mathfrak{g}$-module and $u \circ \alpha$ is an $\mathbf{E}$-valued cocycle on $\mathfrak{g}$, where $u: \operatorname{aff}(\mathbf{E}) \rightarrow \mathbf{E}$ is the translational part (a Lie algebra cocycle on aff $(\mathbf{E})$ ). It is easy to see that conjugating $\alpha$ by a translation does not change the structure of $\mathbf{E}$ as a $\mathfrak{g}$-module but alters $u \circ \alpha$ by a coboundary. Thus the cohomology class of $u \circ \alpha$ in $H^{\mathbf{1}}(\mathfrak{g} ; \mathbf{E})$ is invariant under translational conjugacy and is called the radiance obstruction of $\alpha$, denoted $c_{\alpha} \in H^{1}(\mathfrak{g} ; \mathbf{E})$. Evidently $c_{\alpha}=0$ precisely when there exists $v \in \mathbf{E}$ such that $\alpha(X)(v)=0$ for all $X \in \mathfrak{g}$. In that case $\alpha$ is conjugate (by translation by $v$ ) to a linear representation $\alpha: \mathfrak{g} \rightarrow \operatorname{gl}(\mathbf{E}) \subset \operatorname{aff}(\mathbf{E})$ and we say that $\alpha$ is radiant.

1.4 Let $\alpha: \mathfrak{g} \rightarrow \operatorname{aff}(\mathbf{E})$ be an affine representation. We shall always consider aff $(\mathbf{E})$ as a subalgebra of the Lie algebra $\operatorname{gl}(\mathbf{E} \oplus \mathbf{R})$ using the embedding $\mathbf{J}$ : aff $(\mathbf{E}) \rightarrow$ $\operatorname{gl}(\mathbf{E} \oplus \mathbf{R})$ defined by

$$
\mathbf{J}(X)=\left[\begin{array}{ccc}
\mathbf{L}(X) & & \\
0 & \ldots & 0
\end{array}\right]\left[\begin{array}{c}
u(X) \\
0
\end{array}\right],
$$

where $X \in \operatorname{aff}(\mathbf{E})$. We define $\alpha^{\mathbf{J}}$ to be the composition of $\alpha$ with $\mathbf{J}$. (For the definition of $\alpha^{\mathbf{J}}$ in the case of a group, see [22].)

We shall say that $\alpha$ is reductive if and only if the associated linear representation $\alpha^{\mathbf{J}}: \mathfrak{g} \rightarrow \operatorname{gl}(\mathbf{E} \oplus \mathbf{R})$ is fully reducible. (We make the same definition for affine representations of groups, semigroups, algebras, etc. For example if $\mathfrak{g}$ is semisimple, then any finite-dimensional affine representation is reductive.)

The following lemma is useful and well known (see Milnor [40, 2.3]):

LEMMA. (i) A reductive affine representation is radiant.

(ii) $A$ radiant affine representation $\alpha$ is reductive if and only if its linear part $\mathbf{L} \circ \alpha$ is fully reducible (i.e. reductive).

1.5 Given an affine representation $\alpha: \mathfrak{g} \rightarrow$ aff(E) we will consider the exterior powers $\bigwedge^{k}\left(c_{\alpha}\right) \in H^{k}\left(\mathfrak{g} ; \bigwedge^{k} \mathbf{E}\right)$ of its radiance obstruction. Any pairing $\mathbf{B}: \mathbf{E}_{1} \times$ $\mathbf{E}_{2} \rightarrow \mathbf{E}_{3}$ of $\mathfrak{g}$-modules induces a pairing of the Lie algebra cohomology groups $H^{p}\left(\mathfrak{g} ; \mathbf{E}_{1}\right) \times H^{q}\left(\mathfrak{g} ; \mathbf{E}_{2}\right) \rightarrow H^{p+q}\left(\mathfrak{g} ; \mathbf{E}_{3}\right)$. If $\mathbf{E}$ is a $\mathfrak{g}$-module and $c \in H^{1}(\mathfrak{g} ; \mathbf{E})$ is a cohomology class (i.e. a radiance obstruction of an affine representation), then 
we inductively define $\bigwedge^{n}(c)$ to be the image of $\left(c, \bigwedge^{n-1} c\right)$ under the cohomology pairing $H^{1}(\mathfrak{g} ; \mathbf{E}) \times H^{n-1}\left(\mathfrak{g} ; \bigwedge^{n-1} \mathbf{E}\right) \rightarrow H^{n}\left(\mathfrak{g} ; \bigwedge^{n} \mathbf{E}\right)$ induced by the pairing of $\mathfrak{g}$-modules $\mathbf{E} \times \bigwedge^{n-1} \mathbf{E} \rightarrow \bigwedge^{n} \mathbf{E}$.

1.6 Everything we have said concerning Lie algebras is an infinitesimal version of analogous statements about Lie groups. An alternative approach is to work in the differentiable cohomology of $G$ (which is the same as the continuous cohomology of $G$ ) and then pass to the Lie algebra by differentiating cocycles on $G$. Since it is easier to compute with Lie algebra cohomology, instead we define the radiance obstruction $c_{\alpha}$ of an affine representation $c_{\alpha}$ of a connected Lie group $G$ to be the radiance obstruction of the corresponding affine representation of its Lie algebra (the latter representation being also denoted by $\alpha$ ).

The following basic fact (which motivated this section) illustrates the usefulness of using the cohomology of $\mathfrak{g}$ to linearize questions about $G$ :

THEOREM. Let $\alpha: G \rightarrow \operatorname{Aff}(\mathbf{E})$ be an affine representation of a connected Lie group $G$. Let $c_{\alpha} \in H^{1}(\mathfrak{g} ; \mathbf{E})$ be the radiance obstruction of $\alpha$. If $\alpha(G)$ has an orbit of dimension $\leq k$, then $\bigwedge^{m} c_{\alpha}=0$ for all $m>k$.

PROOF. Since the cohomology class $c_{\alpha}$ is invariant under translational conjugation, we shall assume that the $G$-orbit of 0 has dimension $\leq k$. Let $f_{\alpha}: G \rightarrow \mathbf{E}$ denote the evaluation map of $\alpha$ at 0 , i.e. $f_{\alpha}: g \mapsto \alpha(g)(0)$. Then the differential $d f_{\alpha}: T_{e} G \rightarrow T_{0} \mathbf{E} \cong \mathbf{E}$ satisfies $d f_{\alpha} \circ j=u$, where $u: \mathfrak{g} \rightarrow$ aff $(\mathbf{E})$ is the translational part of $\alpha: \mathfrak{g} \rightarrow \operatorname{aff}(\mathbf{E})$ and $j: \mathfrak{g} \rightarrow T_{e} G$ is the isomorphism defined by restricting left-invariant vector fields on $G$ to the identity $e$.

Since $\operatorname{dim} \alpha(G)(0) \leq k$, the image $u(\mathfrak{g})$ has dimension $\leq k$. Now $\bigwedge^{m}\left(c_{\alpha}\right)$ is represented by the cocycle $\Lambda^{m}(u)$ which takes values in $\Lambda^{m} u(\mathfrak{g})$, which equals zero since $\operatorname{dim} u(\mathfrak{g})=\operatorname{dim} \alpha(G)(0) \leq k<m$. Hence $\bigwedge^{m}(u)=0$ and $\bigwedge^{m}\left(c_{\alpha}\right)$ is zero in $H^{k}\left(\mathfrak{g} ; \bigwedge^{m}(\mathbf{E})\right)$. Q.E.D. E.

1.7 COROLlaRY. If $n=\operatorname{dim} \mathbf{E}$ and $\bigwedge^{n}\left(c_{\alpha}\right) \neq 0$, then $\alpha(G)$ acts transitively on

1.8 Next we shall examine the radiance obstruction and its powers as cohomology classes in a cohomology theory for algebraic groups. The cohomology theory we use is due to Hochschild [29] (see also Mostow [41]), and a main result of [29] reduces its calculation to Lie algebra cohomology.

Let $G$ be a linear algebraic group over $\mathbf{R}$ and suppose that $\rho: G \rightarrow \mathrm{GL}(V)$ is an algebraic representation of $G$ on a real vector space $V$. (In that case we say that $V$ is a rational $G$-module.) Let $\mathfrak{g}$ denote the Lie algebra of $G$. The algebraic cohomology of $G$, denoted $H_{\mathrm{alg}}^{*}\left(G ; V_{\rho}\right)$ (or $H_{\mathrm{alg}}^{*}(G ; V)$ when $\rho$ is understood) is defined to be the cohomology of the complex of Eilenberg-Mac Lane cochains $f: G \times G \times \cdots \times G \rightarrow V$ on $G$ which are regular functions on $G \times G \times \cdots \times G$. It is easy to see that the algebraic cochains form a subcomplex of the analytic (resp. differentiable, continuous, Eilenberg-Mac Lane) cochains on $G$. Moreover the derivative of algebraic cocycle on $G$ at $(e, e, \ldots, e)$ gives a Lie algebra cocycle $\mathfrak{g} \times \mathfrak{g} \times \cdots \times \mathfrak{g} \rightarrow V$, where $V$ is given the induced $\mathfrak{g}$-module structure. In this way there is a natural chain map which induces a cohomology homomorphism $H_{\mathrm{alg}}^{*}(G ; V) \rightarrow H^{*}(\mathfrak{g} ; V)$.

It is well known (see e.g. Humphreys [31]) that every linear algebraic group $G$ decomposes as a semidirect product $U \rtimes R$, where the unipotent radical $U$ is the 
largest unipotent normal subgroup of $G$ and $R$ is a maximal reductive subgroup of $G$ (unique up to conjugacy in $G$ ). We shall denote the unipotent radical of an algebraic group $G$ by $\mathbf{U} G$; it plays an essential role throughout this paper. If $G$ is an algebraic subgroup of $\operatorname{Aff}(\mathbf{E})$, then the reductive subgroup $R$ is a reductive group of affine transformations and is therefore radiant and conjugate in $\operatorname{Aff}(\mathbf{E})$ to a fully reducible linear representation.

The basic result on calculation of algebraic cohomology is due to Hochschild [29] which we summarize as follows:

THEOREM (HOCHSCHILD). Let $G$ be a linear algebraic group over $\mathbf{R}$ and $V$ a rational $G$-module.

(i) If $G$ is unipotent, then $H_{\mathrm{alg}}^{*}(G ; V)$ maps isomorphically onto the Lie algebra cohomology $H^{*}(\mathfrak{g} ; V)$.

(ii) If $G=U \rtimes R$ as above, then the restriction map $H_{\mathrm{alg}}^{*}(G ; V) \rightarrow H_{\mathrm{alg}}^{*}(U ; V)$ is injective and its image is the space of $R$-invariants in $H_{\mathrm{alg}}^{*}(U ; V) \approx H^{*}(U ; V)$, where $\mathcal{U}$ is the Lie algebra of $U$.

1.9 We apply this cohomology theory to affine representations as follows. Let $\mathbf{E}$ be, as usual, a vector space. The group $\operatorname{Aff}(\mathbf{E})$ is a linear algebraic group via the embedding $\mathbf{J}: \operatorname{Aff}(\mathbf{E}) \rightarrow \mathrm{GL}(\mathbf{E} \oplus \mathbf{R})$. The translational part $u: \operatorname{Aff}(\mathbf{E}) \rightarrow \mathbf{E}$ is a regular map which is a cocycle with values in the linear representation $\mathbf{L}: \operatorname{Aff}(\mathbf{E}) \rightarrow \operatorname{GL}(\mathbf{E})$; hence $u$ is an algebraic 1-cocycle on $G$ with values in the $\operatorname{Aff}(\mathbf{E})$-module $\mathbf{E}$. More generally, if $G \subset \operatorname{Aff}(\mathbf{E})$ is an algebraic subgroup, its translational part $u: G \rightarrow \mathbf{E}$ is an algebraic cocycle. We call its cohomology class $[u] \in H_{\text {alg }}^{1}(G ; \mathbf{E})$ the (algebraic) radiance obstruction $c_{G}^{\mathrm{alg}}$ of $G$. By taking exterior powers we obtain algebraic classes $\bigwedge^{k} c_{G}^{\text {alg }} \in H_{\mathrm{alg}}^{k}\left(G ; \bigwedge^{k} \mathbf{E}\right)$. It is clear that under the natural homomorphism $H_{\mathrm{alg}}^{k}\left(G ; \bigwedge^{k} \mathbf{E}\right) \rightarrow H^{k}\left(\mathfrak{g} ; \bigwedge^{k} \mathbf{E}\right)$ the algebraic cohomology class $\bigwedge^{k} c_{G}^{\text {alg }}$ is mapped to the Lie algebra cohomology class $\bigwedge^{k} c_{\mathfrak{g}}$.

THEOREM. Let $G \subset \operatorname{Aff}(\mathbf{E})$ be an algebraic subgroup. Suppose that $\bigwedge^{k} c_{G}^{\mathrm{alg}} \in$ $H_{\mathrm{alg}}^{k}\left(G ; \bigwedge^{k} \mathbf{E}\right)$ is nonzero. Then the unipotent radical $\mathbf{U} G$ cannot have an orbit in $\mathbf{E}$ of dimension $<k$.

(Note that this theorem implies that every orbit of $G$ in $\mathbf{E}$ must have dimension $\geq k$. We have chosen to state this theorem in its stronger form.)

PROOF. By Hochschild's Theorem 1.8(ii) the restriction homomorphism

$$
H_{\mathrm{alg}}^{k}\left(G ; \bigwedge^{k} \mathbf{E}\right) \rightarrow H_{\mathrm{alg}}^{k}\left(\mathbf{U} G ; \bigwedge^{k} \mathbf{E}\right)
$$

is injective; thus $\bigwedge^{k} c_{U G}^{\text {alg }} \neq 0$. By 1.8(i) the associated Lie algebra cohomology class $\bigwedge^{k} c u \neq 0$, where $\mathcal{U}$ is the Lie algebra of $U G$. Now apply 1.6. Q.E.D.

1.10 CoRollary. Let $G \subset \operatorname{Aff}(\mathbf{E})$ be an algebraic subgroup. Suppose $n=$ $\operatorname{dim} \mathbf{E}$. Then $\bigwedge^{n} c_{G}^{\text {alg }} \neq 0$ implies that $G$ acts transitively on $\mathbf{E}$.

In $\S 2$ these results will be applied to compact affine manifolds.

1.11 Equivalent conditions for transitivity of an algebraic affine group. One of the main techniques of this paper is to deduce transitivity of an algebraic subgroup of $\operatorname{Aff}(\mathbf{E})$ from various hypotheses. For this reason we collect here a number of 
conditions on group $\Gamma$ of affine transformations equivalent to the transitivity of its algebraic hull.

A semialgebraic set is a nonempty set defined by finitely many polynomial equations and inequalities; if there are no inequalities, such a set is algebraic, or a variety. Also note that if $G \subset \operatorname{Aff}(\mathbf{E})$ is an algebraic subgroup, then its complexification $G_{\mathbf{C}}$ is naturally contained in the group $\operatorname{Aff}_{\mathbf{C}}(\mathbf{E} \otimes \mathbf{C})$ of complex-affine automorphisms of the complexified vector space $\mathbf{E} \otimes \mathbf{C}$.

THEOREM. Let $\Gamma$ be a subgroup of $\mathrm{Aff}(\mathbf{E})$. Then the following conditions are equivalent:

(a) The algebraic hull $\mathbf{A}(\Gamma)$ of $\Gamma$ acts transitively on $\mathbf{E}$;

(b) The unipotent radical $\mathbf{U A}(\Gamma)$ acts transitively on $\mathbf{E}$;

(c) $\Gamma$ preserves no proper algebraic set;

(d) $\Gamma$ preserves no proper semialgebraic set;

(e) The unipotent radical $\mathbf{U A}(\Gamma)$ of the algebraic hull of $\Gamma$ preserves no proper semialgebraic set;

(f) The complexification $\mathbf{A}_{\mathbf{C}}(\Gamma)$ acts transitively on $\mathbf{E} \otimes \mathbf{C}$;

(g) The complexification $\mathbf{U A}(\Gamma)_{\mathbf{C}}$ of the unipotent radical of $\mathbf{A}(\Gamma)$ acts transitively on $\mathbf{E} \otimes \mathbf{C}$.

Proof. The implications $(\mathrm{b}) \Rightarrow(\mathrm{a}),(\mathrm{b}) \Rightarrow(\mathrm{e}),(\mathrm{d}) \Rightarrow(\mathrm{c})$, and $(\mathrm{g}) \Rightarrow(\mathrm{f})$ are all obvious. We prove $(\mathrm{a}) \Rightarrow(\mathrm{b})$. Decompose $\mathbf{A}(\Gamma)=\mathbf{U A}(\Gamma) \rtimes R$, where $R$ is reductive; thus $R$ fixes a point $x \in \mathbf{E}$. Now $\mathbf{E}=\mathbf{A}(\Gamma) x=\mathbf{U A}(\Gamma) R x=\mathbf{U A}(\Gamma) x$, whence $\mathbf{U A}(\Gamma)$ acts transitively. Thus $(\mathrm{a}) \Rightarrow(\mathrm{b})$. Similarly it follows that (f) $\Rightarrow(\mathrm{g})$.

Next we prove $(\mathrm{a}) \Rightarrow(\mathrm{c})$. Suppose $V$ is a $\Gamma$-invariant proper algebraic set in $\mathbf{E}$. Then $\Gamma$ preserves the ideal $I(V)$ of polynomials vanishing on $V$. The condition $g^{*} I(V)=I(V)$ for $g \in \operatorname{Aff}(\mathbf{E})$ is easily seen to be a polynomial condition on $g$ since $I(V)$ is finitely generated as an ideal and $g$ preserves the filtration of $I(V)$ by degree. Thus if $\Gamma$ preserves $V$, so does $\mathbf{A}(\Gamma)$, contradicting (a). Thus (a) $\Rightarrow(\mathrm{c})$.

Conversely $(\mathrm{c}) \Rightarrow(\mathrm{a})$ because a minimum-dimensional orbit of an algebraic group action is automatically Zariski-closed (see, e.g., Hochschild [30] or Humphreys [31]).

To prove $(\mathrm{c}) \Rightarrow(\mathrm{d})$, let $S \subset E$ be a proper $\Gamma$-invariant semialgebraic set, with frontier (in the Euclidean topology) $\operatorname{fr}(S)=\operatorname{cl}(S)-\operatorname{int}(\operatorname{cl}(S)$ ); note $\operatorname{fr}(S)$ is nonempty. We claim: $\operatorname{fr}(S)$ is contained in a proper algebraic set $V \subset \mathbf{E}$. To this end let $P, Q, R$ be finite sets of nonconstant polynomials $\mathbf{E} \rightarrow \mathbf{R}$ such that $V$ is defined by the conditions $p>0, q \geq 0, r=0$ for $p \in P, q \in Q, r \in R$. If $R$ is nonempty, then take $V$ defined by the equations $r=0$ for $r \in R$. Otherwise, when $R$ is empty, the variety $V$ defined by $V=\bigcup\left\{f^{-1}(0): f \in P \cup Q\right\}$ is easily proved to be proper and to contain $\operatorname{fr}(S)$.

Suppose now that $S$, and thus $\operatorname{fr}(S)$, is $\Gamma$-invariant, and let $V$ be as above. Then $S \subset W=\bigcap\{\gamma V: \gamma \in \Gamma\}$ is a proper and $\Gamma$-invariant algebraic set; therefore (c) $\Rightarrow(\mathrm{d})$.

Next we prove $(\mathrm{e}) \Rightarrow(\mathrm{b})$. Suppose that $\mathbf{U A}(\Gamma)$ does not act transitively on $\mathbf{E}$. By a well-known result of Kostant and Rosenlicht (see Rosenlicht [43] or Hochschild [30]), any orbit of a unipotent algebraic group on an affine variety is Zariski closed. Thus the $\mathbf{U A}(\Gamma)$-orbit of any $x \in \mathbf{E}$ is a $\mathbf{U A}(\Gamma)$-invariant algebraic set, contradicting (e).

Finally we show that $(\mathrm{b}) \Leftrightarrow(\mathrm{g})$. Since the orbits of a unipotent algebraic group are all closed, transitivity of a unipotent action is equivalent to some (and hence all) 
orbits being open. Choose $x \in \mathbf{E}$ and let $f: \mathbf{U A}(\Gamma) \rightarrow \mathbf{E}$ and $f_{\mathbf{C}}: \mathbf{U} \mathbf{A}_{\mathbf{C}}(\Gamma) \rightarrow \mathbf{E} \otimes \mathbf{C}$ be the evaluation maps at $x$ for $\mathbf{U A}(\Gamma)$ and $\mathbf{U} \mathbf{A}_{\mathbf{C}}(\Gamma)$, respectively. Clearly the differential of $f_{\mathbf{C}}$ at $e \in \mathbf{U A}_{\mathbf{C}}(\Gamma)$ is the complexification of the differential of $f$ at $e \in \mathbf{U A}(\Gamma)$. Thus $\mathbf{U A}(\Gamma)$ acts transitively $\Leftrightarrow d f(e)$ is surjective $\Leftrightarrow d f_{\mathbf{C}}(e)$ is surjective $\Leftrightarrow \mathbf{U} \mathbf{A}_{\mathbf{C}}(\Gamma)$ acts transitively on $\mathbf{E} \otimes \mathbf{C}$. The proof of 1.11 is now complete. Q.E.D.

1.12 Dimensions of orbits and powers of the radiance obstruction. Theorems 1.6 and 1.9 show a relation between the dimensions of orbits of an affine action and the maximum nonvanishing power of the radiance obstruction. We may rewrite 1.6 as an inequality

$$
\max \left\{k: \bigwedge^{k} c_{\mathfrak{g}} \neq 0\right\} \leq \min \{\operatorname{dim} G x: x \in \mathbf{E}\}
$$

which relates an algebraic invariant to a geometric invariant of an affine action. A natural question is under what circumstances these two invariants are equal.

The class $c_{\mathfrak{g}}$ is zero precisely when $G$ has a stationary point, i.e. a 0-dimensional orbit. Evidently if either side of the inequality $(*)$ is zero, then both are zero. Moreover, if $G$ has an orbit of dimension $<2$, then $(*)$ is actually equality.

If $G$ possesses a subgroup which acts simply transitively on $\mathbf{E}$, then equality holds in $(*)$, i.e. $\bigwedge^{n} c_{\mathfrak{g}} \neq 0$ where $n=\operatorname{dim} \mathbf{E}$. We shall prove this in two steps. First, when $G$ acts simply transitively, it will be proved in $\S 1$ A. 6 below that $\bigwedge^{n} c_{\mathfrak{g}} \neq 0$. Using this result, we prove that for any group $G$ such that $H \subset G \subset \operatorname{Aff}(\mathbf{E})$, where $H$ acts simply transitively on $\mathbf{E}$, that $\bigwedge^{n} c_{\mathfrak{h}} \neq 0$. For if $\mathfrak{i}: \mathfrak{h} \rightarrow \mathfrak{g}$ is the inclusion of Lie algebras, then $\bigwedge^{n} c_{\mathfrak{g}}=0$ implies $\bigwedge^{n} c_{\mathfrak{h}}=\mathfrak{i}_{*} \bigwedge^{n} c_{\mathfrak{g}}=0$, a contradiction.

Using a similar argument, if $G$ is a connected $k$-dimensional subgroup of a simply transitive affine action $H \subset \operatorname{Aff}(\mathbf{E})$, then equality holds in $(*)$, i.e. $\bigwedge^{k} c_{\mathfrak{g}} \neq 0$; however we shall not need this result.

In general, however, the inequality $(*)$ will be strict, even for transitive unipotent affine actions on $\mathbf{R}^{3}$.

1.13 EXAMPLE. Let $\mathbf{E}=\mathbf{R}^{3}$ and let $\mathfrak{g}$ be the Lie subalgebra of aff( $\left.\mathbf{E}\right)$ consisting of affine maps of the form

$$
\left[\begin{array}{lll}
0 & t & v \\
0 & 0 & u \\
0 & 0 & 0
\end{array}\right]\left[\begin{array}{l}
s \\
t \\
u
\end{array}\right]
$$

The corresponding Lie group $G=\exp (\mathfrak{g})$ comprising affine maps

$$
\left[\begin{array}{ccc}
1 & t & v+t u / 2 \\
0 & 1 & u \\
0 & 0 & 1
\end{array}\right]\left[\begin{array}{l}
\left.s+\left(t^{2}+u v\right) / 2+t u^{2} / 6\right) \\
t+u^{2} / 2 \\
u
\end{array}\right]
$$

acts transitively on $\mathbf{E}$. Let $\sigma, \tau, v, \nu$ denote elements of $\mathfrak{g}^{*}$ corresponding to the variables $s, t, u$, and $v$. As Lie algebra cochains (with real coefficients) we have $d \tau=d v=0, d \nu=\tau \wedge v, d \sigma=v \wedge \nu$. Since $\mathbf{L}(\mathfrak{g}) \subset \operatorname{sl}(\mathbf{E})$, the $\mathfrak{g}$-module $\bigwedge^{3} \mathbf{E}$ is onedimensional with trivial action of $\mathfrak{g}$. Thus the parallel volume form determines an isomorphism $H^{3}\left(\mathfrak{g} ; \bigwedge^{3} \mathbf{E}\right) \approx H^{3}(\mathfrak{g})$. Now a cocycle representing $c_{\mathfrak{g}}$ is the linear map $\mathfrak{g} \rightarrow \mathbf{E}$ defined in coordinates by $(s, t, u, v) \mapsto(s, t, u)$. Thus the 3-cochain $\sigma \wedge \tau \wedge v$ represents the element of $H^{3}(\mathfrak{g})$ corresponding to $\wedge^{3} c_{\mathfrak{g}}$. But $\sigma \wedge \tau \wedge v=d(\sigma \wedge \nu)$ whence $\bigwedge^{3} c_{\mathfrak{g}}=0$. 
We claim $\wedge^{2} c_{\mathfrak{g}} \neq 0$. The exterior 2 -form $\omega=d x \wedge d y+\left(y-z^{2} / 2\right) d y \wedge d z$ is $G$ invariant and pulls back by the evaluation map $G \rightarrow \mathbf{E}$ to an algebraic cohomology class on $G$. Under the Hochschild isomorphism 1.8(i) this cohomology class is represented by the Lie algebra cocycle $\sigma \wedge v$, which is nonzero. Thus

$$
\max \left\{k: \bigwedge^{k} c_{\mathfrak{g}} \neq 0\right\}=2<3=\min \{\operatorname{dim} G x: x \in \mathbf{E}\}
$$

proving that this inequality is sharper than inequality $(*)$ of 1.12 .

\section{A. Appendix: Left-invariant affine structures on Lie groups.}

1A.1 Let $G$ be an $n$-dimensional 1-connected real Lie group with Lie algebra $\mathfrak{g}$ and let $\mathbf{E}=\mathbf{R}^{n}$. An affine structure on $G$ determines a developing map dev: $G \rightarrow \mathbf{E}$, unique up to composition with an element of $\operatorname{Aff}(\mathbf{E})$. This affine immersion serves as local affine coordinates in any open set where it is injective.

1A.2 An affine structure on $G$ is left-invariant if each left-multiplication map $l_{g}: G \rightarrow G(g \in G)$ is an automorphism of the structure. In this case dev determines a unique homomorphism $\alpha: G \rightarrow \operatorname{Aff}(\mathbf{E})$ such that the diagram

$$
\begin{array}{rll}
G & \operatorname{dev} & \mathbf{E} \\
l_{g} \downarrow & \rightarrow & \downarrow \alpha(g) \\
G & \rightarrow & \mathbf{E}
\end{array}
$$

commutes.

Letting $e \in G$ be the identity element, we see that dev coincides with the evaluation map of $\alpha$ at $\operatorname{dev}(e)$. Since $\operatorname{dev}$ is an open map, the developing image $\operatorname{dev}(G)$ is an open set. Evidently $\alpha(G)$ acts transitively on $\operatorname{dev}(G)$, so that $\operatorname{dev}(G)$ is an open orbit of $\alpha(G)$. Since $\operatorname{dim} G=\operatorname{dim} \mathbf{E}$, the isotropy group at any point of $\operatorname{dev}(G)$ is discrete. We shall say that an action is locally simply transitive if there exists an open orbit with discrete isotropy groups. Thus the action $\alpha$ arising from a leftinvariant affine structure is locally simply transitive. Clearly the affine structure on $G$ is complete if and only if $\alpha(G)$ acts simply transitively on $\mathbf{E}$.

Thus to every left-invariant affine structure on $G$ there corresponds an affine action $\alpha$ of $G$ on $\mathbf{E}$ with a distinguished open orbit $\alpha(G) p$ where the action is locally simply transitive. Conversely suppose $\beta: G \rightarrow \operatorname{Aff}(\mathbf{E})$ is an affine representation with $\operatorname{dim} G=\operatorname{dim} \mathbf{E}$ and $p \in \mathbf{E}$ has an open orbit. Then evaluation of $\beta$ at $p$ is an immersion $\mathrm{ev}_{p}: G \rightarrow \mathbf{E}$ and the action $\beta$ is locally simply transitive. The unique affine structure on $G$ for which $\mathrm{ev}_{p}$ is a developing map is left-invariant (because $\beta$ is a homomorphism to $\operatorname{Aff}(\mathbf{E})$ ).

1A.3 Thus we may identify left-invariant affine structures on a Lie group $G$ with pairs $(\alpha, \Omega)$, where $\alpha$ is a locally simply transitive affine action of $G$ and $\Omega$ is an open orbit. Two left-invariant affine structures are isomorphic if and only if the corresponding representations are conjugate in $\mathrm{Aff}(\mathbf{E})$ by a conjugacy taking one orbit to another.

Fix a left-invariant affine structure on $G$ and a corresponding pair $(\alpha, \Omega)$. Commutativity of the diagram above shows that left-invariant tensor fields on $G$ correspond bijectively via $\operatorname{dev}$ to $\alpha(G)$-invariant tensor fields on the open orbit $U$. In particular $G$ has a left-invariant parallel volume form if and only if $\alpha(G)$ is contained in the special affine group $\operatorname{SAff}(\mathbf{E})$ comprising all volume-preserving affine automorphisms of E. Similarly $G$ has a left-invariant radiant vector field if and only if $\alpha(G)$ fixes a point of $\mathbf{E}$. 
If $\Gamma \subset G$ is a discrete subgroup, then the space $\Gamma \backslash G$ of right cosets inherits an affine structure from the left-invariant affine structure on $G$ and dev: $G \rightarrow \mathbf{E}$ is a developing map for the affine manifold $\Gamma \backslash G$. Any left-invariant tensor field on $G$ descends to a left-invariant tensor field on $\Gamma \backslash G$.

Let $G$ be a Lie group with a left-invariant affine structure. By abuse of language we shall say that $G$ is radiant (respectively $G$ has parallel volume) in case $G$ has a left-invariant parallel volume form (resp. has a left-invariant radiant vector field).

If $\alpha: G \rightarrow \operatorname{Aff}(\mathbf{E})$ is an affine representation which is locally simply transitive at $x \in \mathbf{E}$, then the associated affine representation of Lie algebras, also denoted $\alpha: \mathfrak{g} \rightarrow$ aff $(\mathbf{E})$, has the property that its translational part at $x$, given by $Y \mapsto \alpha(Y)(x)$, is a linear isomorphism $\mathfrak{g} \rightarrow \mathbf{E}$. The image $\alpha(\mathfrak{g})$ consists of affine vector fields on $\mathbf{E}$. These vector fields integrate to give the action of $G$ on $\mathbf{E}$ corresponding under dev to left-multiplication; thus these vector fields correspond to right-invariant vector fields on $G$. In summary: on a Lie group with left-invariant affine structure, the rightinvariant vector fields are affine vector fields. (On the other hand, left-invariant vector fields are generally not even polynomial vector fields; see [13] .)

1A.4 Unlike affine structures on compact manifolds, it is possible for a leftinvariant affine structure on a Lie group to both be radiant and have parallel volume. Here is the simplest example. Let $G_{1}$ be the subgroup of $\operatorname{SL}(2, \mathbf{R})$ comprising matrices of the form

$$
\left[\begin{array}{ll}
e^{s} & t \\
0 & e^{-s}
\end{array}\right]
$$

Then $G_{1}$ acts simply transitively on the half-plane $\left\{(x, y) \in \mathbf{R}^{2}: y>0\right\}$ and therefore inherits a left-invariant affine structure, which is both radiant and volumepreserving.

This example also shows that in contrast to affine structures on compact manifolds, where it is conjectured that parallel volume implies completeness, it is possible for a left-invariant affine structure to have parallel volume and be incomplete. In the other direction, a left-invariant affine structure which is complete does not necessarily have parallel volume. Let $G_{2}$ be the subgroup of $\operatorname{Aff}\left(\mathbf{R}^{2}\right)$ consisting of affine maps of the form

$$
\left[\begin{array}{cc}
e^{s} & 0 \\
0 & 1
\end{array}\right]\left[\begin{array}{l}
t \\
s
\end{array}\right] .
$$

Then $G_{2}$ acts simply transitively on the entire plane and therefore the corresponding left-invariant affine structure is complete; however every $G_{2}$-invariant area form on $\mathbf{R}^{2}$ must be a constant multiple of $e^{-y} d x \wedge d y$ so the left-invariant structure does not have parallel volume.

For more examples and a more thorough discussion of left-invariant affine structures on Lie groups, the reader is referred to Auslander [1], Boyom [4], Fried [10], Fried and Goldman [11], Fried, Goldman and Hirsch [13], Helmstetter [25, 26], Kim [56], Medina [38, 39], Milnor [40], and Vinberg [53].

1A.5 The following theorem is due to J. Helmstetter [26]:

THEOREM. Let $G$ be a Lie group with left-invariant affine structure. Then the affine structure is complete if and only if right-invariant volume forms are parallel 
COROLlaRY. Suppose $G$ is a unimodular Lie group with left-invariant affine structure. Then this structure is complete if and only if it has parallel volume.

We shall give one and a half proofs of Helmstetter's theorem: one proof of the "if" assertion based on 1.6, and a more geometric version of Helmstetter's original proof.

1A.6 Suppose that $G$ is a Lie group with left-invariant affine structure. Using 1.6, we show that if right-invariant volume forms are parallel, then $G$ is complete. We may assume that $\alpha: G \rightarrow \operatorname{Aff}(\mathbf{E})$ is the affine representation corresponding to left-multiplication and that $\alpha$ is locally simply transitive at the origin 0 . Let $\omega_{\mathbf{E}}$ denote a parallel volume form on $\mathbf{E}$; the hypothesis that "right-invariant volume forms are parallel" is equivalent to the assertion that the volume form $\omega_{G}=\operatorname{dev}^{*} \omega_{\mathbf{E}}$ is right-invariant on $G$. We must show that $G$ acts simply transitively on $\mathbf{E}$. Since $G$ already acts locally simply transitively on $\mathbf{E}$, it will suffice to show that $G$ acts transitively on E. By 1.6 it is enough to show that the top power $\bigwedge^{n} c_{\mathfrak{g}}$ of the radiance obstruction is nonzero, where $n=\operatorname{dim} G$.

The proof that $\bigwedge^{n} c_{\mathfrak{g}} \neq 0$ uses the classical work of Koszul [33] on Lie algebra cohomology. Koszul defines the analog of a fundamental cohomology class in Lie algebra cohomology. Let $n=\operatorname{dim} \mathfrak{g}$ and consider the $\mathfrak{g}$-module $\bigwedge^{n} \mathfrak{g}$ given by the top exterior power of the adjoint representation. It is easy to see that this module is one-dimensional and the representation of $\mathfrak{g}$ on it is given by the modular representation $\lambda: \mathfrak{g} \rightarrow \mathbf{R}, \lambda(X)=\operatorname{tr} \operatorname{ad}(X)$. We shall denote this one-dimensional $\mathfrak{g}$-module by $\mathbf{R}_{\lambda}$. Koszul proved that the cohomology $H^{n}\left(\mathfrak{g} ; \mathbf{R}_{\lambda}\right) \cong \mathbf{R}$. (Koszul goes on to use a generator of this group to define a Poincaré duality in $H^{*}(\mathfrak{g})$ but we shall not need this.) Just as elements of $\Lambda^{n} \mathfrak{g}^{*}$ correspond to left-invariant volume forms on $G$, elements of $\Lambda^{n} \mathfrak{g}^{*} \otimes \mathbf{R}_{\lambda}$ correspond to right-invariant volume forms on $G$. Koszul's theorem asserts that a right-invariant volume form has a nonzero cohomology class when suitably interpreted as a left-invariant form in a certain line bundle.

To show that $\Lambda^{n} c_{\mathfrak{g}}$ is nonzero, consider the pairing $H^{0}\left(\mathfrak{g} ; \bigwedge^{n} \mathbf{E}^{*} \otimes \mathbf{R}_{\lambda}\right) \times$ $H^{n}\left(\mathfrak{g} ; \bigwedge^{n} \mathbf{E}\right) \rightarrow H^{n}\left(\mathfrak{g} ; \mathbf{R}_{\lambda}\right)$ induced by the coefficient pairing $\left(\bigwedge^{n} \mathbf{E}^{*} \otimes \mathbf{R}\right)_{\lambda} \times$ $\bigwedge^{n} \mathbf{E} \rightarrow \mathbf{R}_{\lambda}$. Then the image of $\left(\omega_{\mathbf{E}}, \bigwedge^{n} c_{\mathfrak{g}}\right)$ is the cohomology class of the pullback $\omega_{G}$ in $H^{n}\left(\mathfrak{g} ; \mathbf{R}_{\lambda}\right)$. By Koszul's theorem, this class is nonzero; hence $\bigwedge^{n} c_{\mathfrak{g}} \neq 0$, whence $G$ acts transitively. Thus $G$ has a complete affine structure.

1A.7 One defect of this proof is that it gives no information in the case that the affine structure is incomplete. In Helmstetter's original proof Theorem 1A.5 is deduced from a result showing a close relationship between the growth of volume under right-multiplication and the geometry of the frontier of the developing image. Our efforts to understand Helmstetter's proof led us to the following geometric discussion.

Our geometric approach to Helmstetter's theorems begins with the representation of right-invariant vector fields as affine vector fields. Choose a basis $X_{1}, \ldots, X_{n}$ $\epsilon \alpha(\mathfrak{g})$ of affine vector fields on $\mathbf{E}$ representing a basis of right-invariant vector fields on $G$. Then the exterior product $\mu=X_{1} \wedge \cdots \wedge X_{n}$ is a polynomial exterior $n$-vector field which represents a right-invariant volume current on $G$. Thus we may write $\mu=f(x) \partial / \partial x_{1} \wedge \cdots \wedge \partial / \partial x_{n}$ where $f: \mathbf{E} \rightarrow \mathbf{R}$ is a polynomial of degree $\leq n$ in the affine coordinates $\left(x_{1}, \ldots, x_{n}\right)$ of $\mathbf{E}$. It follows that any right-invariant volume form on $G$ is a constant multiple of $f(x)^{-1} d x_{1} \wedge \cdots \wedge d x_{n}$. 
The following theorem is Helmstetter's main result from which he deduces 1A.5:

1A.8 THEOREM. The developing map dev: $G \rightarrow \mathbf{E}$ is a covering map onto a connected component of the set $\{x \in \mathbf{E}: f(x) \neq 0\}$.

Proof. Let $g_{G}$ be a right-invariant Riemannian metric on $G$. Since $g_{G}$ is invariant under the transitive action of $G$ on itself by right-multiplications, the metric $g_{G}$ is complete. Furthermore there is a symmetric 2-form $g_{\mathbf{E}}$ on $\mathbf{E}$ with rational coefficients such that $g_{G}=\operatorname{dev}^{*} g_{\mathbf{E}}$. For let $X_{1}, \ldots, X_{n}$ be an orthonormal basis of right-invariant vector fields; then the cometric $g_{\mathbf{E}}^{*}$ dual to $g_{\mathbf{E}}$ is the tensor $\left(X_{1}\right)^{2}+\cdots+\left(X_{n}\right)^{2}$ which is polynomial of degree $\leq 2$ in affine coordinates, i.e.

$$
g_{\mathbf{E}}^{*}=\sum_{i, j=1}^{n} g^{i j}(x) \partial / \partial x_{i} \partial / \partial x_{j},
$$

where each $g^{i j}(x)$ is polynomial of degree $\leq 2$. Moreover $\operatorname{det}\left[g^{i j}(x)\right]=f(x)^{2}$; and the matrix representing $g_{\mathbf{E}}$ is

$$
\left[g_{i j}(x)\right]=\left[g_{i j}(x)\right]^{-1}=\operatorname{det}\left[g_{i j}(x)\right]^{-1} \operatorname{adj}\left[g^{i j}(x)\right]
$$

and has rational entries. Specifically, the symmetric 2-form $f(x)^{2} g_{\mathbf{E}}(x)$ is a polynomial tensor field. Thus a right-invariant Riemannian metric on a Lie group with left-invariant affine structure must be rational (compare [20]).

The developing map dev: $G \rightarrow \mathbf{E}$ is a local isometry from $G$ to the open subset $\Omega$ of $\mathbf{E}$ where $g_{\mathbf{E}}$ is Riemannian metric. Since $g_{G}$ is complete, dev is a covering map onto a connected component of $\Omega$. Since the tensor field $f(x)^{2} g_{\mathbf{E}}(x)$ is polynomial, it is everywhere defined. It follows that if $x \in \partial \omega$, then either $g_{\mathbf{E}}$ blows up at $x$ or $g_{\mathbf{E}}(x)$ is degenerate. In either case $f(x)=0$. Thus dev is a covering map onto a connected component of $\{x \in \mathbf{E}: f(x) \neq 0\}$. This completes the proof of 1A.8.

1 A.9 To deduce 1A.5 from 1 A. 8 we proceed as follows. We first show that right-invariant parallel volume forms $\Rightarrow$ completeness. Observe that right-invariant volume forms are parallel if and only if $f(x)$ is constant. By 1A.8 it follows that $\operatorname{dev}$ is a covering map (and hence a diffeomorphism) onto $\mathbf{E}$. Hence $G$ is complete.

In the converse direction it will be useful to complexify. Namely if $\alpha: G \rightarrow$ $\operatorname{Aff}(\mathbf{E})$ is locally simply transitive at $0 \in \mathbf{E}$, then the complexified representation $\alpha_{\mathbf{C}}: G_{\mathbf{C}} \rightarrow \operatorname{Aff}_{\mathbf{C}}(\mathbf{E} \otimes \mathbf{C})$ is also locally simply transitive at 0 (see 1.13). Suppose $G$ acts simply transitively on $\mathbf{E}$. Then by $1.11, G_{\mathbf{C}}$ acts transitively on $\mathbf{E} \otimes \mathbf{C}$. In particular $G_{\mathbf{C}}$ must act simply transitively on $\mathbf{E} \otimes \mathbf{C}$. Applying $1 \mathrm{~A} .8$ to the induced complete left-invariant complex affine structure on $G_{\mathbf{C}}$, we see that the polynomial $f_{\mathbf{C}}: \mathbf{E} \otimes \mathbf{C} \rightarrow \mathbf{C}$ has no zeros, and hence is constant. The proof of 1A.5 is complete. Q.E.D.

\section{The algebraic hull of the holonomy group of a compact affine man- ifold.}

2.1 Let $M$ be a compact affine manifold with a fixed universal covering $p: \tilde{M} \rightarrow$ $M$, and let $\pi=\pi_{1}(M)$ be the group of deck transformations. Let dev: $\tilde{M} \rightarrow \mathbf{E}$ be a fixed developing map with holonomy homomorphism $h: \pi \rightarrow \operatorname{Aff}(\mathbf{E})$. Let $\Gamma=h(\pi) \subset \operatorname{Aff}(\mathbf{E})$ be the affine holonomy group and $\mathbf{A}(\Gamma)$ its algebraic huil in $\operatorname{Aff}(\mathbf{E})$. We denote the unipotent radical of $\mathbf{A}(\Gamma)$ by $\mathbf{U A}(\Gamma)$.

The main result of this section applies Theorem 1.6 to affine structures on compact manifolds. 
2.2 The affine tangent bundle $T^{\mathrm{aff}}(M)$ of $M$ (as described in Goldman and Hirsch $[\mathbf{2 2}])$ is classified by a sequence of maps:

$$
M \rightarrow B \pi \rightarrow B \Gamma \rightarrow B \operatorname{Aff}(\mathbf{E})^{\delta} \rightarrow B \operatorname{Aff}(\mathbf{E}) .
$$

(Here $\operatorname{Aff}(\mathbf{E})^{\delta}$ denotes the group $\operatorname{Aff}(\mathbf{E})$ with the discrete topology.) $T^{\text {aff }}(M)$ is classified as a topologically flat bundle by the first map, as a $\Gamma$-bundle by the next map, then as a flat affine bundle, and finally as an affine bundle by the last map.

2.3 THEOREM (2.6 OF [22]). Let $M$ be a compact affine manifold which possesses a parallel $k$-form $\omega$ which has nonzero cohomology class $[\omega] \in H^{k}(M ; \mathbf{R})$. Then $\bigwedge^{k} c_{M} \neq 0$.

Combining this theorem with 1.6, we obtain

2.4 THEOREM. Let $M$ be a compact affine manifold which possesses a parallel $k$-form which has nonzero cohomology class in $H^{k}(M: \mathbf{R})$. Then every orbit of $\mathbf{U A}(\Gamma)$ has an orbit of dimension $\geq k$.

2.5 COROLLARY. Let $M$ be a compact affine manifold with parallel volume. Then $\mathbf{A}(\Gamma)$ acts transitively.

(Observe that the transitivity of $\mathbf{A}(\Gamma)$ is equivalent to the transitivity of $\mathbf{U A}(\Gamma)$ and many other algebraic and geometric conditions; see 1.11.)

In $§ 2.7$ Corollary 2.5 will be used to prove several cases of the main conjecture (that parallel volume $\Leftrightarrow$ completeness for compact affine manifolds). Before exploring the consequences of 2.5 , we observe that its conclusion is valid under the (conjecturally equivalent) hypothesis of completeness.

2.6 THEOREM. Let $M$ be a compact complete affine manifold. Then $\mathbf{A}(\Gamma)$ acts transitively on $\mathbf{E}$.

Proof. Decompose $\mathbf{A}(\Gamma)$ as a semidirect product $\mathbf{U A}(\Gamma) \rtimes R$ of its unipotent radical $\mathbf{U A}(\Gamma)$ and a maximal reductive subgroup $R$. It suffices to show that $\mathbf{U A}(\Gamma)$ acts transitively. The group $R$, being reductive, fixes a point $b \in \mathbf{E}$. Since $\mathbf{A}(\Gamma)=R \mathbf{U A}(\Gamma)=\mathbf{U A}(\Gamma) R$, the $\mathbf{U A}(\Gamma)$-orbit $\mathbf{U A}(\Gamma) b$ is invariant under $\mathbf{A}(\Gamma)$. In particular it is invariant under $\Gamma$.

Let $G=\mathbf{U A}(\Gamma)$ and let $H \subset G$ be the isotropy subgroup at $b$. Since $G$ and $H$ are unipotent algebraic groups, the exponential map induces a homeomorphism $\mathfrak{g} / \mathfrak{h} \rightarrow G / H$. Therefore $\mathbf{U A}(\Gamma) / H$ is contractible.

The map $f: \mathbf{U A}(\Gamma) / H \rightarrow \mathbf{E}$ defined by $g H \mapsto g b$ is injective. Since $\Gamma$ acts freely and properly discontinuously on $\mathbf{E}$, its restriction to the orbit $\mathbf{U A}(\Gamma) b$ is also free and properly discontinuous. The map $\mathbf{U A}(\Gamma) \rightarrow \mathbf{E}$ is $\Gamma$-equivariant. There is a commutative diagram:

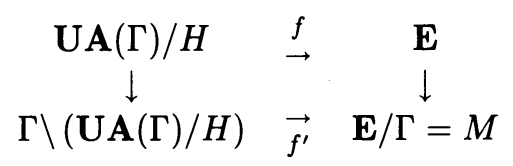

The vertical arrows are covering maps and the map $f$ induces an isomorphism on fundamental groups. Since $\mathbf{U A}(\Gamma) / H$ and $\mathbf{E}$ are both contractible, it follows that $f^{\prime}$ is a homotopy equivalence. Since both $\Gamma \backslash(\mathbf{U A}(\Gamma) / H)$ and $M$ are manifolds and $M$ is compact, $f^{\prime}$ is surjective. It follows that $\operatorname{dim} \mathbf{U A}(\Gamma) b=\operatorname{dim} \mathbf{E}$. 
Since every orbit of a connected unipotent affine action is closed (see Hochschild $[\mathbf{3 0}]$, Rosenlicht $[\mathbf{4 3}])$, the orbit $\mathbf{U A}(\Gamma) b$ is open and closed in $\mathbf{E}$ and thus equals E. Hence $\mathbf{U A}(\Gamma)$, and equivalently $\mathbf{A}(\Gamma)$, acts transitively on E. Q.E.D.

2.7 Now we deduce a few immediate corollaries of the transitivity of the algebraic hull.

PROPOSITION. Let $M$ be a compact affine manifold having parallel volume. Then the developing image $\operatorname{dev}(\tilde{M}) \subset \mathbf{E}$ is not a proper semialgebraic set.

Proof. By $2.5 \mathbf{A}(\Gamma)$ acts transitively on $\mathbf{E}$. By 1.11 it follows that $\Gamma$ preserves no proper semialgebraic set. Since $\operatorname{dev}(\tilde{M})$ is $\Gamma$-invariant, the proposition follows. Q.E.D.

2.8 COROLlARY. Suppose that $M$ is a compact affine manifold satisfying the hypothesis

$(* *) \quad \operatorname{dev}: \tilde{M} \rightarrow \mathbf{E}$ is a covering map onto a semialgebraic open set.

If $M$ has parallel volume, then $M$ is complete.

2.9 REMARKS. (a) We do not know if there exists a compact incomplete affine manifold whose developing map is surjective. On the other hand there are many examples of compact affine manifolds whose developing maps are not coverings. (See Smillie $[45,47]$, Sullivan and Thurston [49], and Goldman $[14,18]$ for these examples.) For example the product of any surface of genus $\geq 1$ with a circle admits an affine structure whose developing map is not a covering map. Indeed if the genus is $>1$, then affine structures exist whose developing images are the complement of the origin in $\mathbf{R}^{3}$ and whose holonomy is a dense subgroup of $\operatorname{GL}(3 ; \mathbf{R}) . \operatorname{In}[\mathbf{1 3}]$ it is shown that if $M$ is a compact incomplete affine manifold with nilpotent holonomy, then the developing map is not surjective.

(b) In general the developing image is quite far from being semialgebraic; in fact the boundary of $\operatorname{dev}(\tilde{M})$ need not be smooth or even rectifiable. For example there exist convex cones $\Omega \subset \mathbf{R}^{3}$ covering compact affine 3-manifolds (homeomorphic to a product of a surface with a circle) whose boundaries are not $C^{2}$ (Kac and Vinberg [32], Goldman [14]). In dimension 4, there are domains in $\mathbf{R}^{4}=\mathbf{C}^{2}$ with nonrectifiable boundary, covering complex affine structures on $S^{1} \times T_{1}(F)$, where $F$ is a compact surface of genus $>1$. (Both of these types of structures are affine structures induced from projective structures; see Sullivan and Thurston [49], Benzecri [31], or Goldman $[\mathbf{1 4}, \mathbf{1 8}]$.) There are examples where the developing images are bounded by cones on circle bundles over limit sets of Kleinian groups. See Sullivan and Thurston [49] for more details.

2.10 THEOREM. Let $M$ be a connected compact affine manifold. Assume that $M$ is complete or has parallel volume. Then:

(i) $M$ admits no nonconstant rational function $M \rightarrow \mathbf{R}$.

(ii) Every rational tensor field of type $(p, q)$ on $M$ is polynomial of degree $\leq$ $(p+q) A_{n}$, where $A_{n}=(2 n-2) ! /\left(2^{n-1}(n-1) !\right)$. 
Proof. (i) Let $f: M \rightarrow \mathbf{R}$ be a rational function on $M$. There exists a rational function $F: \tilde{M} \rightarrow \mathbf{R}$ such that the diagram

$$
\begin{array}{ccc}
\tilde{M} & f & \mathbf{E} \\
p \downarrow & \rightarrow & \downarrow \\
M & \vec{F} & \mathbf{R}
\end{array}
$$

commutes. The Euclidean closure of each level set $F^{-1}(c)$ which meets $\operatorname{dev}(\tilde{M})$ is a $\Gamma$-invariant proper algebraic subset; this set is also $\mathbf{A}(\Gamma)$-invariant. It follows from 2.5-2.6 and 1.11, however, that $\mathbf{A}(\Gamma)$ acts transitively on $\mathbf{E}$; so this is impossible. Q.E.D.

(ii) Let $\omega_{M}$ be a rational $(p, q)$-tensor on $M$. There is a unique rational $(p, q)$ tensor $\omega_{\mathbf{E}}$ such that $\operatorname{dev}^{*}\left(\omega_{\mathbf{E}}\right)=\omega$ projects to $\omega_{M}$. Since $\omega_{\mathbf{E}}$ is rational it is $\mathbf{U A}(\Gamma)$-invariant. Since $\mathbf{U A}(\Gamma)$ acts transitively on $\mathbf{E}$ by $2.5-2.6$ it suffices to prove the following:

2.11 LEMMA. Let $G \subset \operatorname{Aff}(\mathbf{E})$ be a unipotent subgroup acting transitively on E. Then every $G$-invariant tensor field $\omega$ of type $(p, q)$ is polynomial of degree $\leq(p+q) A_{n}$.

ProOF. Let $\mathfrak{g} \subset$ aff(E) be the Lie algebra of $G$ and let $\mathfrak{h}$ be the Lie algebra of the isotropy group $H=G \cap \mathrm{GL}(\mathbf{E})$. Let $A$ be any linear subspace complementary to $\mathfrak{h}$ in $\mathfrak{g}$, i.e. $\mathfrak{h} \oplus \mathfrak{A}=\mathfrak{g}$. Recall that a polynomial isomorphism of vector spaces is a polynomial mapping $f: \mathbf{E}_{1} \rightarrow \mathbf{E}_{2}$ which is bijective and such that $f^{-1}$ is polynomial. The proof of the following lemma is identical to the proof in the case $G$ simply transitive (Theorem 8.3 of $[\mathbf{1 3}]$ ) and is therefore omitted.

2.12 LEMMA. The composition $A \hookrightarrow \mathfrak{g} \stackrel{\exp }{\rightarrow} G \stackrel{u}{\rightarrow} \mathbf{E}$ is a polynomial isomorphism $A \rightarrow \mathbf{E}$ of degree $\leq A_{n}$.

By Lemma 2.12, $\omega(x)=(\exp f(x))_{\#} \omega_{0}$, where $f: \mathbf{E} \rightarrow \mathcal{A}$ is the inverse to $u \circ$ exp: $\mathcal{A} \rightarrow \mathbf{E}$ and $g_{\#}$ denotes the map induced on the tensor algebra by $g \in G$. Thus $\omega: \mathbf{E} \rightarrow\{$ tensors on $\mathbf{E}\}$ is a composition of polynomial maps and is polynomial. Since $\operatorname{deg} f \leq A_{n}$ it follows that $\operatorname{deg} \omega \leq(p+q) A_{n}$. Q.E.D.

2.13 Homogeneous affine structures. Let $M$ be an affine manifold and let $\operatorname{Aff}(M)$ denote the group of affine automorphisms $M \rightarrow M$. Then $\operatorname{Aff}(M)$ is a Lie group of diffeomorphisms of $M$. We say $M$ is homogeneous if $\operatorname{Aff}(M)$ acts transitively on $M$. For example, a Lie group with left-invariant affine structure is a homogeneous affine manifold. Homogeneous affine manifolds have been studied by Koszul [34, 35], Matsushima [37], Shima [44], Yagi [54] and others.

We shall prove the main conjecture for compact homogeneous affine manifolds:

THEOREM. Let $M$ be a compact homogeneous affine manifold. Then $M$ is complete if and only if $M$ has parallel volume.

2.14 The first step in the proof of Theorem 2.13 is the following lemma:

LEMMA. Let $M$ be a homogeneous complete affine manifold. Then the affine holonomy $\Gamma$ is unipotent and $M$ has parallel volume.

Proof. Since $M$ is complete, $M \approx \mathbf{E} / \Gamma$ where $\Gamma \subset \operatorname{Aff}(\mathbf{E})$ is a discrete subgroup. $\operatorname{Aff}(M)$ is identified with $N / \Gamma$ where $N \subset \operatorname{Aff}(\mathbf{E})$ is the normalizer of $\Gamma$. 
Since $\Gamma$ is discrete the identity component $N^{0}$ centralizes $\Gamma$. It is easy to see that homogeneity of $M$ implies that $N^{0}$ is transitive on $\mathbf{E}$. Let $\gamma \in \Gamma$ be any element. Since $\gamma$ is a deck transformation it has no fixed point, which implies that 1 is an eigenvalue of the linear part of $\gamma$. Therefore the Fitting component of $\gamma$ is nontrivial. (The Fitting component is the largest $\gamma$-invariant affine subspace upon which $\gamma$ acts unipotently.) The centralizer of $\gamma$ in $\operatorname{Aff}(\mathbf{E})$ preserves $\mathbf{E}_{U}$; therefore $\mathbf{E}_{U}$ is invariant under $N^{0}$. But $N^{0}$ is transitive, so $\mathbf{E}_{U}=\mathbf{E}$. Q.E.D.

REMARKS. The same proof shows that if $M$ is a complete affine manifold such that the centralizer of $\Gamma$ in $\operatorname{Aff}(\mathbf{E})$ leaves invariant no proper affine subspace (i.e. $\Gamma$ acts irreducibly on $\mathbf{E}$ ), then $\Gamma$ is unipotent. Using a similar technique to the proof of 2.14, Auslander [1] proves that the group $\operatorname{Aff}(M)^{0}$, where $M$ is as above, is a nilpotent Lie group.

2.15 Proposition. Let $M$ be a homogeneous affine manifold. Then its developing map is a covering onto a semialgebraic open set.

Theorem 2.13 follows from Proposition 2.15, together with Corollary 2.8.

PROOF OF PROPOSITION 2.15. Pass to the universal covering space of $M$ in order to assume that $M$ has a homogeneous affine structure induced by an immersion dev: $M \rightarrow \mathbf{E}$ (any covering space of a homogeneous affine manifold is still homogeneous). If $g \in \operatorname{Aff}(M)$ then $g \circ \operatorname{dev}$ is another developing map for $M$ so there exists $\varphi(g) \in \operatorname{Aff}(\mathbf{E})$ so that dev $\circ g=\varphi(g) \circ$ dev. It is easy to see that $\varphi: \operatorname{Aff}(M) \rightarrow \operatorname{Aff}(\mathbf{E})$ is a homomorphism with respect to which dev is equivariant. Since $\operatorname{Aff}(M)$ acts transitively on $M$, the $\operatorname{Aff}(M)$-equivariant immersion dev is a covering map onto its image. The developing image $\operatorname{dev}(M)$ is an open orbit of the identity component $G$ of $\varphi(\operatorname{Aff}(M))$ and for any connected subgroup $G \subset \operatorname{Aff}(\mathbf{E})$, the union of open $G$-orbits is a Zariski-open subset of $\mathbf{E}$. (For a proof of this fact, let $\mathfrak{g} \subset$ aff(E) be the Lie algebra of $G$ and for $X \in \mathfrak{g}$ let $\mathbf{L}(X)$ and $u(X)$ be the linear part and the translational part of $X$, respectively. Then the differential of the evaluation map of $G$ at $y \in \mathbf{E}$ is the linear map $u_{y}: \mathfrak{g} \rightarrow \mathbf{E}$ given by $X \mapsto$ $\mathbf{L}(X) y+u(X)$. The condition that $y \in \mathbf{E}$ have $G$-orbit of dimension $\leq k$ is precisely the condition that $u_{y}$ have rank $\leq k$. This is evidently a polynomial condition on $y$.) Thus the union of open $G$-orbits in $\mathbf{E}$ is Zariski open and in particular, since a connected component of a Zariski open set is semialgebraic, any one open $G$-orbit is semialgebraic. (Indeed, a connected component of a semialgebraic set is itself semialgebraic; one may prove this using the existence of semialgebraic triangulations of semialgebraic sets (see Hironaka [58]) although more elementary proofs are available.) It follows that $\operatorname{dev}: \tilde{M} \rightarrow \mathbf{E}$ is a covering map onto an open semialgebraic set. Q.E.D.

2.16 Rational Riemanniar metrics. Let $M$ be an affine manifold. A rational Riemannian metric on $M$ is a Riemannian metric (defined everywhere on $M$ ) whose coefficients in local affine coordinates are locally defined rational functions. For further discussion and examples of rational Riemannian metrics, see Goldman and Hirsch [20].

THEOREM. Let $M$ be a compact affine manifold with a rational Riemannian metric $g$. The following conditions are equivalent:

(a) $M$ is complete;

(b) $M$ has parallel volume; 
(c) $g$ is a polynomial Riemannian metric;

(d) $M$ is finitely covered by a complete affine nilmanifold.

ProOF. Suppose $g_{M}$ is a rational Riemannian metric on $M$. There exists a $\Gamma$-invariant rational 2-tensor $g_{\mathbf{E}}$ on $\mathbf{E}$ such that $\operatorname{dev}^{*} g_{\mathbf{E}}=p^{*} g_{M}$ (where $p: \tilde{M} \rightarrow M$ is the projection). Evidently $g_{\mathbf{E}}$ is invariant under $\mathbf{A}(\Gamma)$, because $g_{\mathbf{E}}$ is $\mathfrak{g}$-invariant and rational. Since $\mathbf{A}(\Gamma)$ acts transitively on $\mathbf{E}$, it follows by 2.10 (ii) that $g$ is polynomial. Thus $(\mathrm{a}) \Rightarrow(\mathrm{c})$.

Since $g_{\mathbf{E}}$ is defined on a Zariski open subset $\Omega \subset \mathbf{E}$, it follows from Proposition 3 of [20] that dev: $M \rightarrow \Omega$ is a covering onto a connected component $\Omega_{0}$. By $2.15, \Omega_{0}$ is semialgebraic; therefore 2.8 shows that $(\mathrm{b}) \Rightarrow(\mathrm{a})$. By [13, Theorem 8.4], $(\mathrm{d}) \Rightarrow(\mathrm{a})$. By [20, Theorem 1], (c) $\Leftrightarrow(\mathrm{d})$; and $(\mathrm{d}) \Rightarrow(\mathrm{a})$ is clear. Q.E.D.

\section{Parallel volume and completeness for affine manifolds with solvable holonomy.}

3.1 One obtains interesting classes of affine manifolds by imposing algebraic assumptions on the affine holonomy group $\Gamma$. A natural assumption is that $\Gamma$ be solvable, owing to the abundance of examples: all known complete affine manifolds have virtually solvable holonomy group. Many interesting compact incomplete affine manifolds have solvable holonomy; see $[\mathbf{1 3}, \mathbf{1 6}, \mathbf{1 9}]$ for examples.

The equivalence of parallel volume and completeness was proved in $[\mathbf{1 3}]$ for compact affine manifolds with nilpotent holonomy; and it immediately follows for virtually nilpotent holonomy as well. We prove that for manifolds with virtually solvable holonomy, completeness $\Rightarrow$ parallel volume, but we know only partial results in the other direction.

3.2 THEOREM. Let $M$ be a compact complete affine manifold. Suppose that $\pi=\pi_{1}(M)$ is virtually solvable. Then $M$ has parallel volume.

ProOF. By $[\mathbf{1 1}, 1.5], M$ has a finite covering $\hat{M}$ which is affinely equivalent to a complete affine solvmanifold, i.e. an affine manifold of the form $\Gamma \backslash G$ where $G$ is a Lie group with left-invariant complete affine structure and $\Gamma \subset G$ a lattice subgroup. Equivalently $\hat{M}=\mathbf{E} / \Gamma$ where $\Gamma \subset G \subset \operatorname{Aff}(\mathbf{E})$ with $G$ acting simply transitively on $\mathbf{E}$. Since $G$ admits a lattice, it is unimodular, so by Corollary 1A.5 $G$ is volume-preserving. Thus $\Gamma$ is volume-preserving and $\hat{M}$ has parallel volume. It follows that $M$ itself has parallel volume. Q.E.D.

3.3 COROLlaRY. Let $M$ be a compact 3-dimensional affine manifold which is complete. Then $M$ has parallel volume.

PROOF. The proof follows from 3.2 and $[\mathbf{1 1}, 2.12]$ where it is shown that $\pi_{1}(M)$ is virtually solvable. Q.E.D.

3.4 Now we turn to the converse statement. Let $\Gamma$ be a solvable group and consider the derived series $\Gamma=\Gamma_{1} \triangleright \Gamma_{2} \triangleright \cdots \triangleright \Gamma_{s}=\{1\}$, where $\Gamma_{k+1}$ is the derived group of $\Gamma_{k}$. The rank of $\Gamma$ is defined to equal the sum

$$
\sum_{i=1}^{s-1} \operatorname{dim}_{\mathbf{R}}\left(\mathbf{R} \otimes \Gamma_{i} / \Gamma_{i+1}\right)
$$

which may be infinite (even if $\Gamma$ is finitely generated). If $\Gamma$ is polycyclic (see Milnor [40] or Raghunathan [42] for basic properties of polycyclic groups), then each $\Gamma_{i}$ 
is finitely generated and $\operatorname{rank}(\Gamma)$ is finite. Furthermore $\operatorname{rank}(\Gamma)$ equals the rank of $\Gamma$ as a polycyclic group, which is the virtual cohomological dimension of $\Gamma$. Solvable holonomy groups of complete affine manifolds are known to be polycyclic, but Smillie (unpublished) has constructed compact affine manifolds with solvable but not virtually polycyclic holonomy.

3.5 THEOREM. Let $M$ be a compact affine manifold with affine holonomy group $\Gamma \subset \operatorname{Aff}(\mathbf{E})$ which is solvable and $\operatorname{rank}(\Gamma) \leq \operatorname{dim} M$. If $M$ has parallel volume, then $M$ is complete.

REMARK. It follows from $[\mathbf{1 1}, \S 1]$, that $M$ is finitely covered by a solvmanifold. The condition $\operatorname{rank}(\Gamma) \leq \operatorname{dim} M$ implies that the affine holonomy homomorphism $\pi \rightarrow \operatorname{Aff}(\mathbf{E})$ factors through a solvable Lie group of dimension $\leq \operatorname{dim} M$.

3.6 The following lemma is proved in $[\mathbf{1 1}, \S 1]$ (which is based upon Raghunathan $[42,4.28])$.

LEMMA. Let $\rho: \Gamma \rightarrow \mathrm{GL}(\mathbf{E})$ be a linear representation of a solvable group $\Gamma$. There exists a solvable subgroup $G \subset \mathrm{GL}(V)$ having finitely many connected components such that $\rho(\Gamma) \subset G \subset \mathbf{A}(\Gamma)$ and $\operatorname{dim}(G) \leq \operatorname{rank}(\Gamma)$.

3.7 Proof of 3.5. Apply Lemma 3.6 to the inclusion $\Gamma \subset \mathbf{A}(\Gamma)$ to obtain a subgroup $G$ with $\Gamma \subset G \subset \mathbf{A}(\Gamma)$ with $\operatorname{dim} G \leq \operatorname{rank}(\Gamma) \leq \operatorname{dim}(\mathbf{E})$. We claim that $G \subset \operatorname{Aff}(\mathbf{E})$ acts simply transitively on $\mathbf{E}$.

Decompose $\mathbf{A}(\Gamma)=\mathbf{A}(G)$ in the usual way, $\mathbf{A}(\Gamma)=\mathbf{U A}(\Gamma) \rtimes R$, where $R$ is reductive. Let $\Psi: \mathbf{A}(\Gamma) \rightarrow R$ be the projection homomorphism and define the projection map $\Phi: \mathbf{A}(\Gamma) \rightarrow \mathbf{U A}(\Gamma)$ by $g \mapsto g^{-1} \Psi(g)$. One can prove (following Raghunathan [42, Theorem 4.28]) that for a connected solvable Lie group $G$ consisting of matrices, $\Phi$ maps $G$ onto $\mathbf{U A}(G)$. Since $\mathbf{U A}(\Gamma)=\mathbf{U A}(G)$, we see that $\Phi$ maps $G$ onto $\mathbf{U A}(\Gamma)$.

Since $M$ is compact with parallel volume, 2.5 implies that $\mathbf{U A}(\Gamma)$ acts transitively. We now show $G$ acts transitively: let $y \in \mathbf{E}$ be a fixed point of the reductive group $R$; since $\Phi(g) y=g^{-1} y$ for $g \in G, G y=\mathbf{U A}(\Gamma) y=\mathbf{E}$. It follows that $\operatorname{dim} G=\operatorname{dim} \mathbf{E}$. Since $\mathbf{E}$ is simply connected, each isotropy group is trivial. Thus $G$ acts simply transitively.

It is easy to see that $M$ must now be complete. For example, we may find a $G$-invariant, and hence $\Gamma$-invariant, Riemannian metric $g_{\mathbf{E}}$ on $\mathbf{E}$ which defines a Riemannian metric $g_{M}$ which is complete. Since dev: $\tilde{M} \rightarrow \mathbf{E}$ is a local isometry between $p^{*} g_{M}$ and $g_{\mathrm{E}}$, it is a covering map onto $\mathbf{E}$. Therefore $M$ is complete. Q.E.D.

3.8 COROLLARY. Let $M$ be a compact affine manifold which is aspherical and has virtually polycyclic fundamental group (for example, if $M$ is homeomorphic to a solvmanifold). Then $M$ is complete if and only if it has parallel volume.

ProOF. The hypotheses on $M$ imply that $\pi$ is virtually polycyclic of rank equal to $\operatorname{dim}$ E. 3.8 follows immediately from 3.2 and 3.5. Q.E.D.

There are many interesting examples of compact affine manifolds with solvable holonomy which are convex, i.e. dev: $M \rightarrow \mathbf{E}$ is a bijection onto a convex open subset of $\mathbf{E}$; see Koszul $[\mathbf{3 4}, \mathbf{3 5}]$, Goldman [16], and Vey $[\mathbf{5 0}, \mathbf{5 1}, \mathbf{5 2}]$ for discussion. 3.8 implies that parallel volume is equivalent to completeness for this class of affine structures. 
3.9 COROLLARY. Let $M^{3}$ be a compact 3-dimensional affine manifold with virtually solvable fundamental group. Then $M$ is complete if and only if it has parallel volume.

PROOF. It is proved in Evans and Moser $[8]$ that $\pi_{1}(M)$ is virtually solvable of rank $\leq 3$. Now apply 3.2 and 3.5. Q.E.D.

\section{Affine manifolds with nilpotent holonomy.}

4.1 In what follows $M$ will be a compact affine manifold whose affine holonomy group $\Gamma$ is nilpotent. The class of affine manifolds with nilpotent holonomy is a rich class of structures (see $[\mathbf{1 3}]$ and Smillie $[\mathbf{4 5}, \mathbf{4 7}, \mathbf{4 8}]$ ). The equivalence of parallel volume and completeness is proved in $[\mathbf{1 3}]$, as well as several other conditions for this class of manifolds. Combining this with results proved above we obtain

THEOREM. Let $M$ be a compact $n$-dimensional affine manifold with nilpotent affine holonomy group $\Gamma \subset \operatorname{Aff}(\mathbf{E})$ and developing map dev: $\tilde{M} \rightarrow \mathbf{E}$. The following conditions are equivalent:

(a) $M$ is complete;

(b) $M$ has parallel volume;

(c) dev is surjective;

(d) the affine action of $\Gamma$ on $\mathbf{E}$ is irreducible;

(e) $\Gamma$ is unipotent;

(f) $M$ is a complete affine nilmanifold;

(g) $\bigwedge^{n} c_{M} \neq 0$;

(h) $\mathbf{A}(\Gamma)$ acts transitively.

Proof. The equivalence of (a), (b), (c), (d), (e), and (f) was proved in [13]. The implication $(\mathrm{b}) \Rightarrow(\mathrm{g})$ (which holds without nilpotence) follows from Theorem 2.3 , and $(\mathrm{g}) \Rightarrow(\mathrm{h})$ by 1.10 . We prove the contrapositive of $(\mathrm{h}) \Rightarrow(\mathrm{d})$. Suppose that $\Gamma$ is reducible, that is, $\Gamma$ leaves invariant an affine subspace $F \subset \mathbf{E}$. The affine subspace $F$ is $\mathbf{A}(\Gamma)$-invariant, contradicting transitivity of $\mathbf{A}(\Gamma)$. Q.E.D.

4.2 The main result of this section is that inequality $(*)$ of 1.12 is an equality for the algebraic hull of a nilpotent holonomy group of a compact affine manifold:

THEOREM. Let $M$ be a compact affine manifold with nilpotent affine holonomy group $\Gamma \subset \operatorname{Aff}(\mathbf{E})$. Then the largest power $k$ such that $\bigwedge^{k} c_{M}$ is nonzero equals the minimum dimension of an $\mathbf{A}(\Gamma)$-orbit in $\mathbf{E}$. Furthermore if $M$ is incomplete, the unique $k$-dimensional orbit lies outside the developing image.

We conjecture that Theorem 4.2 holds more generally, e.g. for compact affine manifolds with solvable holonomy.

4.3 We shall deduce 4.2 by showing that the Fitting subspace of the affine holonomy is the minimum-dimensional $\mathbf{A}(\Gamma)$-orbit, and a parallel volume on the Fitting subspace has nonzero cohomology class. Much of the proofs of these facts are contained in $[\mathbf{1 3}]$ and our joint work with G. Levitt [23]. We summarize the results from these papers which we need as follows:

PROPOSITION. Let $M$ be a compact affine manifold with nilpotent holonomy $\Gamma \subset \operatorname{Aff}(\mathbf{E})$.

(i) There exists a unique $\Gamma$-invariant affine subspace $\mathbf{E}_{U} \subset \mathbf{E}$ upon which $\Gamma$ acts unipotently and a unique affine projection $\pi: \mathbf{E} \rightarrow \mathbf{E}_{U}$ commuting with $\Gamma$. 
(ii) $\pi \circ \operatorname{dev}: \tilde{M} \rightarrow \mathbf{E}_{U}$ is a surjective fibration and $\mathbf{E}_{U}$ is disjoint from the developing image.

(iii) There exists $\gamma \in \Gamma$ whose linear part $\mathbf{L}(\gamma)$ restricted to a fiber of $\pi$ is an expansion.

4.4 The affine subspace $\mathbf{E}_{U}$ is called the Fitting subspace. Let $k=\operatorname{dim} \mathbf{E}_{U}$. Since $\Gamma$ acts unipotently on $\mathbf{E}_{U}$ it preserves a parallel volume form $\omega_{U}$ on $\mathbf{E}_{U}$. The pullback $\omega_{\mathbf{E}}=\pi^{*} \omega_{U}$ is a $\Gamma$-invariant parallel $k$-form on $\mathbf{E}$. Hence it defines a parallel (and hence closed) $k$-form $\omega_{M}$ on $M$.

Proposition. The cohomology class $\left[\omega_{M}\right] \in H^{k}(M ; \mathbf{R})$ is nonzero.

For the proof see Goldman, Hirsch and Levitt [23]. The following corollary states that the dimension of the Fitting subspace $\mathbf{E}_{U}$ is the maximum exponent of a nonvanishing power of the radiance obstruction:

4.5 COROLlaRY. $\Lambda^{k} c_{M}$ is a nonzero cohomology class in $H^{k}\left(M ; \Lambda^{k} \mathbf{E}\right)$, but $\bigwedge^{k+1} c_{M}$ is zero in $H^{k+1}\left(M ; \bigwedge^{k} \mathbf{E}\right)$, where $k=\operatorname{dim} \mathbf{E}_{U}$.

ProOF OF 4.5. That $\bigwedge^{k} c_{M} \neq 0$ follows from 2.3. That $\bigwedge^{k+1} c_{M}=0$ follows from 1.6. Q.E.D.

4.6 We shall deduce that the Fitting subspace $\mathbf{E}_{U}$ is a minimum-dimensional orbit of $\mathbf{A}(\Gamma)$ from the following:

THEOREM. (i) $\mathbf{A}(\Gamma)$ acts transitively on $\mathbf{E}_{U}$;

(ii) Every $\Gamma$-invariant Zariski-closed nonempty subset of $\mathbf{E}$ contains $\mathbf{E}_{U}$.

Since an $\mathbf{A}(\Gamma)$-orbit of minimum dimension is Zariski-closed, 4.6 implies that the Fitting subspace is an $\mathbf{A}(\Gamma)$-orbit of minimum dimension. Thus for the proof of 4.2 it suffices to prove 4.6.

4.7 The proof of 4.6(i) is based on the notion of a syndetic action. We shall say that a group $G$ acts syndetically on a space $X$ if and only if there exists a compact $K \subset X$ with $G K=X$. If $M$ is a compact affine manifold, then the affine holonomy action on the developing image is syndetic. If $M$ has nilpotent holonomy $\Gamma$, then $\Gamma$ acts syndetically on $\mathbf{E}_{U}$ because $\pi: \operatorname{dev}(\tilde{M}) \rightarrow \mathbf{E}_{U}$ is surjective $[\mathbf{1 3}, 6.9]$. Furthermore since the algebraic hull of $\Gamma \mid \mathbf{E}_{U}$ is the restriction of $\mathbf{A}(\Gamma)$ to $\mathbf{E}_{U}, 4.6(\mathrm{i})$ follows from

LEMMA. Let $G \subset \operatorname{Aff}(\mathbf{E})$ act unipotently and syndetically on $\mathbf{E}$. Then $\mathbf{A}(G)$ acts transitively on $\mathbf{E}$.

Proof OF LemMA 4.7. If $G$ is unipotent then so is $\mathbf{A}(G)$. If $G$ acts syndetically so does any group containing $G$. Thus we may replace $G$ by $\mathbf{A}(G)$ and assume $G$ is an algebraic unipotent subgroup of $\operatorname{Aff}(\mathbf{E})$. We must show that $G$ acts transitively.

If $\operatorname{dim} \mathbf{E}=1$, then $G$ is a nontrivial group of translations so $\mathbf{A}(G)$ is the full group of translations. Hence the lemma is true when $\mathbf{E}$ is 1-dimensional.

Inductively assume that 4.7 has been proved for $\mathbf{E}$ having dimension less than $n$. Suppose that $\mathbf{E}$ has dimension $n$ and $G \subset \operatorname{Aff}(\mathbf{E})$ acts unipotently and syndetically. Since $G$ acts unipotently it preserves a parallel 1-form, i.e. there exists a linear functional $\eta: \mathbf{E} \rightarrow \mathbf{R}$ such that for each $g \in G$, the expression $v(g)=\eta(g(x))-\eta(x)$ is 
independent of $x \in \mathbf{E}$. One sees easily that $v: G \rightarrow \mathbf{R}$ is a homomorphism. Furthermore $v: G \rightarrow \mathbf{R}$ is surjective: for otherwise $\eta$ would be $G$-invariant, contradicting syndeticity. The kernel Ker $v=G_{1}$ is a subgroup which acts on the hyperplane $\mathbf{E}_{1}=\eta^{-1}(0)$. Choose a right-inverse $\mu: \mathbf{R} \rightarrow G$ to $v: G \rightarrow \mathbf{R}$.

Let $K$ be a compact fundamental set for the action of $G$ on $\mathbf{E}$, i.e. $\mathbf{E}=G K$. Let $K_{1}=\bigcup\left\{\mu(t) K \cap \mathbf{E}_{1}: t \in \mathbf{R}\right\}$. We show that $K_{1}$ is compact and $G_{1} K_{1}=\mathbf{E}_{1}$. Since $K$ is compact the set of $t$ such that $K$ intersects $\mu(-t) \mathbf{E}_{1}$ is a compact set of real numbers; thus the set of all $t \in \mathbf{R}$ such that $\mu(t) K \cap \mathbf{E}_{1}$ is nonempty is compact. Hence $K_{1}$ is compact. Furthermore $G_{1} K_{1}=G_{1} \mu(\mathbf{R}) K \cap \mathbf{E}_{1}=G K \cap \mathbf{E}_{1}=\mathbf{E}_{1}$. Therefore $G_{1}$ acts syndetically on $\mathbf{E}_{1}$ with fundamental set $K_{1}$.

By the induction hypothesis, $\mathbf{A}\left(G_{1}\right)$ acts transitively on each $\eta^{-1}(p)$. There is a commutative diagram:

$$
\begin{array}{lll}
\mathbf{E} & \stackrel{\eta}{ } & \mathbf{R} \\
\downarrow & & \downarrow \\
\mathbf{E} / G & \overrightarrow{\hat{\eta}} & \mathbf{R} / v(G)
\end{array}
$$

Since $\eta$ is surjective, $\hat{\eta}$ is surjective, implying that $\mathbf{R} / v(G)$ is compact. Hence $\mathbf{A}(v(G))$ acts transitively on $\mathbf{R}$. Now the exact sequence $G_{1} \rightarrow G \rightarrow \mathbf{R}$ determines an exact sequence

$$
\mathbf{A}\left(G_{1}\right) \rightarrow \mathbf{A}(G) \rightarrow \mathbf{A}(v(G)) .
$$

Since $\mathbf{A}\left(G_{1}\right)$ acts transitively on $v^{-1}(p)$ and $\mathbf{A}(v(G))$ acts transitively on $\mathbf{R}$, it follows $\mathbf{A}(G)$ acts transitively on $\mathbf{E}$. Q.E.D.

ProOF OF LEMMA 4.6(ii). Let $V \subset \mathbf{E}$ be a $\Gamma$-invariant subset. Then being Zariski-closed, $V$ is $\mathbf{A}(\Gamma)$-invariant. Write $\mathbf{E}=\mathbf{E}_{U} \oplus F$, where $F=\operatorname{Ker} \pi: \mathbf{E} \rightarrow \mathbf{E}_{U}$. By 4.3(iii) there exists $\gamma \in \Gamma$ such that $\mathbf{L}(\gamma)$ restricted to $F$ is a linear expansion. Let $\gamma^{(s)}$ be the semisimple part of $\gamma$; by $[\mathbf{3 1}], \gamma^{(s)} \in \mathbf{A}(\Gamma)$. Then $\mathbf{E}_{U}$ is the stationary set of $\gamma^{(s)}$ and $\gamma^{(s)}$ preserves each coset of $F$ in $\mathbf{E}$.

Choose a coset $x+F$ which intersects $V$, where $x \in \mathbf{E}_{U}$. Then $V \cap(x+F)$ is a closed subset of $x+F$ invariant under $\gamma^{(s)}$. Since for every $y \in(x+\mathbf{E}), \gamma^{n} y \rightarrow$ $x \in \mathbf{E}_{U}$, we have $x \in V$. Since $\mathbf{A}(\Gamma)$ acts transitively on $\mathbf{E}_{U}, \mathbf{A}(\Gamma) x \in \mathbf{E}_{U}$. Since $V$ is $\mathbf{A}(\Gamma)$ - invariant, $V \supset \mathbf{E}_{U}$. Q.E.D.

4.8 We have seen that a compact affine manifold with nilpotent holonomy $\Gamma$ has associated with it a certain unipotent affine action, namely the restriction of $\Gamma$ to the Fitting subspace. We call this unipotent action the Fitting component of the affine holonomy action. It is natural to ask for criteria that a unipotent affine action be a Fitting component for the holonomy of a compact affine manifold with nilpotent holonomy. Obvious necessary conditions are that $G$ be finitely generated and that $G$ act syndetically on $\mathbf{E}$. By combining 1.13 with 4.2 we see that these conditions are not sufficient:

THEOREM. Let $M$ be a compact affine manifold with nilpotent holonomy $\Gamma \subset$ $\operatorname{Aff}(\mathbf{E})$. Suppose that the Fitting subspace $\mathbf{E}_{U}$ is three-dimensional. Then the action of $\Gamma$ restricted to $\mathbf{E}_{U}$ cannot lie in the group of all affine transformations of the form

$$
\left[\begin{array}{ccc}
1 & t-u^{2} / 2 & v \\
0 & 1 & u \\
0 & 0 & 1
\end{array}\right]\left[\begin{array}{l}
s \\
t \\
u
\end{array}\right]
$$


Proof. By 4.2, $\bigwedge^{3} c_{M} \neq 0$. But $\bigwedge^{3} c_{M}$ is the image of $\bigwedge^{3} c_{\Gamma}$ under $H^{3}\left(\Gamma ; \bigwedge^{3} \mathbf{E}\right)$ $\rightarrow H^{3}\left(M ; \bigwedge^{3} \mathbf{E}\right)$ and $\bigwedge^{3} c_{\Gamma}$ is the image of $\bigwedge^{3} c_{G}$ under the map $H^{3}\left(G ; \bigwedge^{3} \mathbf{E}\right)$ $\rightarrow H^{3}\left(\Gamma ; \bigwedge^{3} \mathbf{E}\right)$ induced by restriction. However $\bigwedge^{3} c_{G}=0$ by 1.13 . This contradiction proves 4.8 .

\section{REFERENCES}

1. L. Auslander, Simply transitive groups of affine motions, Amer. J. Math. 99 (1977), 809821.

2. J. P. Benzecri, Variétés localement affines, Thèse, Princeton Univ., Princeton, N.J., 1955.

3. __ Variétés localement affines et projectives, Bull. Soc. Math. France 88 (1960), 229332.

4. N. Boyom, Structures affines sur les groupes de Lie nilopotents (preprint).

5. G. Bredon, Sheaf theory, McGraw-Hill, New York, 1967.

6. H. Cartan and S. Eilenberg, Homological algebra, Princeton Univ. Press, Princeton, N.J., 1956.

7. C. Chevalley and S. Eilenberg, Cohomology theory of Lie groups and Lie algebras, Trans. Amer. Math. Soc. 63 (1948), 85-124.

8. B. Evans and L. Moser, Solvable fundamental groups of compact 3-manifolds, Trans. Amer. Math. Soc. 168 (1972), 189-210.

9. D. Fried, Polynomial on affine manifolds, Trans. Amer. Math. Soc. 274 (1982), 709-719.

10. _ Distality, completeness, and affine structures, J. Differential Geom. (to appear).

11. D. Fried and W. Goldman, Three-dimensional affine crystallographic groups, Adv. in Math. 47 (1983), 1-49.

12. D. Fried, W. Goldman and M. W. Hirsch, Affine manifolds and solvable groups, Bull. Amer. Math. Soc. (N.S.) 3 (1980), 1045-1047.

13. _ Affine manifolds with nilpotent holonomy, Comment. Math. Helv. 56 (1981), 487523.

14. W. Goldman, Affine manifolds and projective geometry on surfaces, Senior Thesis, Princeton Univ., Princeton, N.J., 1977.

15. , Discontinuous groups and the Euler class, Doctoral Dissertation, Univ. of California, Berkeley, 1980.

16. _ Two examples of affine manifolds, Pacific J. Math. 94 (1981), 327-330.

17. __ On the polynomial cohomology of affine manifolds, Invent. Math 65 (1982), 453457.

18. __ Projective structures with Fuchsian holonomy (submitted).

19. , (in preparation).

20. W. Goldman and M. W. Hirsch, A generalization of Bieberbach's theorem, Invent. Math. 65 (1981), 1-11.

21. __ Polynomial forms on affine manifolds, Pacific J. Math 101 (1982), 115-121.

22. Math. Soc. 286 (1984), 629-649.

23. W. Goldman, M. W. Hirsch and G. Levitt, Invariant measures for affine foliations, Proc. Amer. Math. Soc. 86 (1982), 511-518.

24. A. Haefliger, Differentiable cohomology, C.I.M.E. lectures, Varenna, Italy, 1979.

25. J. Helmstetter, Algèbres symétriques à gauche, C. R. Acad. Sci. Paris 272 (1971), 10881091.

26. _ـ Radical d'une algèbre symétrique à gauche, Ann. Inst. Fourier (Grenoble) 29 (1979), 17-35.

27. M. W. Hirsch, Flat manifolds and the cohomology of groups, Algebraic and Geometric Topology, Lecture Notes in Math., vol. 664, Springer-Verlag, New York, 1977.

28. M. W. Hirsch and W. P. Thurston, Foliated bundles, flat manifolds, and invariant mea sures, Ann. of Math. 101 (1975), 369-390.

29. G. Hochschild, Cohomology of algebraic linear groups, Illinois J. Math. 5 (1961), 492-519.

30. __ Basic theory of algebraic groups and Lie algebras, Graduate Texts in Math., vol. 75, Springer-Verlag, Berlin, Heidelberg and New York, 1981. 
31. J. Humphreys, Linear algebraic groups, Graduate Texts in Math., vol. 21, Springer-Verlag, Berlin, Heidelberg and New York, 1975.

32. V. Kac and E. B. Vinberg, Quasi-homogeneous cones, Math. Notes 1 (1967), 231-235 (translated from Math. Zametki 1 (1967), 347-354).

33. J. L. Koszul, Homologie et cohomologie des algèbres de Lie, Bull. Soc. Math. France 78 (1950), 66-127.

34. __ Domaines bornés homogènes et orbites des groupes de transformations affine, Bull. Soc. Math. France 89 (1961), 515-533.

35. __ Ouvertes convexes homogènes des espaces affine, Math. Z. 139 (1974), 254-259.

36. L. Markus, Cosmological models in differential geometry, mimeographed notes, Univ. of Minnesota, 1962, p. 58.

37. Y. Matsushima, Affine structures on complex manifolds, Osaka J. Math. 5 (1968), 215-222.

38. A. Medina Perea, Flat left-invariant connections adapted to the automorphism structure of a Lie group, J. Differential Geometry 16 (1981), 445-474.

39.

40. J. W. Milnor, On fundamental groups of complete affinely flat manifolds, Adv. in Math. 25 (1977), 178-187.

41. G. D. Mostow, Cohomology of topological groups and solvmanifolds, Ann. of Math. 73 (1961), 20-48.

42. M. S. Raghunathan, Discrete subgroups of Lie groups, Ergebnisse der Math., Band 68, Springer-Verlag, Berlin, Heidelberg and New York, 1972.

43. M. Rosenlicht, On quotient varieties and the embedding of certain homogeneous spaces, Trans. Amer. Math. Soc. 101 (1961), 211-233.

44. H. Shima, Homogeneous Hessian manifolds, Manifolds and Lie Groups, Papers in honor of Yozo Matsushima, Progress in Math., vol. 14, Birkhäuser, Boston, Mass., 1981, pp. 385-392.

45. J. Smillie, Affinely flat manifolds, Doctoral Dissertation, Univ. of Chicago, 1977.

46. __ An obstruction to the existence of affinely flat manifolds, Invent. Math. 64 (1981), 411-415.

47. _ Affine structures with diagonal holonomy, I.A.S. preprint (1979).

48. Complex affine manifolds with nilpotent holonomy (in preparation).

49. D. Sullivan and W. Thurston, Manifolds with canonical coordinates: some examples, Enseign. Math. 29 (1983), 15-25.

50. J. Vey, Sur une notion d'hyperbolicité des variétés localement plates, These, Univ. de Grenoble, 1969.

51. __ Sur une notion d'hyperbolicité des variétés localement plates, C. R. Acad. Sci. Paris 266 (1968), 622-624.

52. _ Sur les automorphismes affines des ouverts convexes saillants, Ann. Scuola Norm. Sup. Pisa 4 (1970), 641-665.

53. E. B. Vinberg, Homogeneous convex cones, Trans. Moscow Math. Soc. 12 (1963), 340-403.

54. K. Yagi, Hessian structures on affine manifolds, Manifolds and Lie Groups, Papers in honor of Yozo Matsushima, Progress in Math., vol. 14, Birkhäuser, Boston, Mass., 1981.

55. - On compact homogeneous affine manifolds, Osaka J. Math. 7 (1970), 457-475.

56. H. Kim, On complete left-invariant affine structures on 4-dimensional nilpotent Lie groups, Thesis, Univ. of Michigan, 1983.

57. H. Shima, On certain locally flat homogeneous manifolds of solvable Lie groups, Osaka J. Math. 13 (1976), 213-229.

58. H. Hironaka, Triangulations of algebraic sets, Algebraic Geometry, Arcata 1974, Proc. Sympos. Pure Math., vol. 29, Amer. Math. Soc., Providence, R.I., 1975, pp. 165-186.

\section{Mathematical Sciences Research Institute, Berkeley, California 94720}

DePaRTMENT OF MATHEMATICS, 2-281, MASSACHUSETtS INSTITUTE OF TEChNOLOGY, CAMBRIDGE, MASSACHUSETTS 02139 (Current address of W. M. Goldman)

DePaRtMENT OF MATHEMATICS, UNIVERSity of CALIFORNIA, BERKELEy, CALIFORNIA 94720 (Current address of M. W. Hirsch) 